\title{
Nobody's Perfect; Matched Layers for Heterogeneous Media
}

\author{
Laurence Halpern $^{*} \quad$ Ludovic Métivier ${ }^{\dagger} \quad$ Jeffrey Rauch ${ }^{\ddagger} \quad$ Juliette Ryan ${ }^{\S}$
}

\begin{abstract}
We present an example of Bérenger's method for a problem with variable coefficients at the interface showing that the method is not perfectly matched. The reflections are revealed by constructing an infinitely accurate geometric optics approximation. They are also revealed in numerical simulations that confirm the dependence on wavelength from the asymptotic expansions.
\end{abstract}

\section{Introduction}

This paper analyses reflections at artificial absorbing layers used at the external part of computational domains. They are used to truncate to finite size computations aimed at computing solutions on infinite or very large domains. Particular attention is paid to Bérenger's layers $[8,9,10]$ that under a variety of situations are perfectly matched, that is give rise to no reflections at all. We give a natural example where this method is not perfectly matched when coefficients are not constant on a neighborhood of the interface.

Computing reflections for variable coefficient problems requires care. The standard method freezes coefficients, drops lower order terms and reasons with plane waves. It is a good way to compute the leading reflection term. For intelligent absorbing boundaries including Bérenger's method the leading term is equal to zero. The plane wave computation does not suffice to evaluate the small reflections that are produced. We compute infinitely accurate high frequency asymptotic solutions. The imperfection is revealed by the presence of non zero reflected waves in the high order correctors. The order at which these waves appear in the expansion is a measure of the effectiveness of the absorbing layer.

We analyse a wave equation that is a close cousin to the acoustic equations of geoscience. In addition to the accurate high frequency solutions, we perform numerical simulations. The size of the reflections as a function of frequency matches the prediction of geometric optics.

The asymptotic expansions give a tool to compare imperfect strategies. Indeed we begin by computing reflections for methods simpler than Bérenger's. In order to avoid amplifying layers that sometimes are created by Bérenger's layers [6], [16], smart layers were introduced in the latter article. They are dissipative by elementary energy identities. Even with variable coefficients. In this article we introduce a refinement called tuned layers that are tuned to a presumably known wave number. They are dissipative and reflect at

*LAGA, UMR 7539 CNRS, Université Paris 13, 93430 Villetaneuse, FRANCE, halpern@math.univparis13.fr

${ }^{\dagger}$ LJK, CNRS, Univ. Grenoble Alpes, Grenoble, France. ludovic.metivier@ujf-grenoble.fr

${ }^{\ddagger}$ Department of Mathematics, University of Michigan, Ann Arbor 48109 MI, USA rauch@umich.edu

$\S$ ONERA, Chemin de la Hunière, 91123 PALAISEAU, France. Juliette.Ryan@onera.fr 
the same order as Bérenger's method. On our test problem the imperfect Bérenger problem outperforms the other two methods tested. Had we tested a problem where the Bérenger layers were amplifying, the performances would have been reversed as in [23], [15]. The tuned layers are never amplifying.

There is very little analysis in the literature for time dependent Bérenger methods when there are variable coefficients at the transition to the layer. Variable coefficients also arise when analysing constant coefficient problems in polar coordinates using circular computational domains and annular absorbing layers [27], [12]. Relying on the analyticity of the coefficients, these authors show that the method of complex coordinate deformation yields perfection in the time harmonic case. For truly time dependent problems it is not clear what conclusion to draw as different temporal components would require distinct deformations.

Collino and Tsogka [13] find reflections in numerical simulations using Bérenger's method in heterogeneous media. They see large reflections from the external boundary of the computational domain. Their computations may also show the small reflections from the imperfection at the transition to the layer.

Bérenger's strategy is one of many approaches to the construction of weakly reflecting absorbing layers. Our main result reveals a limit to its effectiveness. We neither summarize, evaluate, nor criticize other strategies. The interested reader can consult [21], [14], [5], [18], [25], [19], [17], [1], [2], [7], [4], [3], [22], [24], [20], listed here in chronological order, to appreciate the variety of interesting ideas and methods.

Consider the hyperbolic operator

$$
L_{1}\left(\partial_{t}, \partial_{x}\right):=\partial_{t}+\sum_{j=1}^{d} A_{j}(x) \partial_{j}, \quad \partial_{j}:=\frac{\partial}{\partial x_{j}}, \quad 1 \leq j \leq d .
$$

The coefficients $A_{j}$ are $N \times N$ matrix valued. The computational domain $\left.\mathcal{D}:=\prod_{j=1}^{d}\right]-\ell_{j}, \ell_{j}[$ contains a not too much smaller rectangle $\mathcal{R}$ that includes the domain of interest and the support of the data. The $2^{d}$ hyperplanes defining the faces of the boundary of $\mathcal{R}$ form a network in $\mathcal{D}$ denoted $\mathcal{N}$. The goal is to modify the equation in the layer $\mathcal{D} \backslash \overline{\mathcal{R}}$ so that solutions are both weakly reflected at the interfaces comprising $\mathcal{N}$ and are damped in the layers comprising $\mathcal{D} \backslash \overline{\mathcal{R}}$.

In one situation it is easy to construct perfectly matched absorbing layers. If the coefficient matrices $A_{j}$ are real diagonal and invertible, then the system consists of $N$ uncoupled scalar transport equations with nonvanishing velocity in the $x_{j}$ direction. It is then easy to place perfectly absorbing boundaries and also perfectly nonreflective layers at an artificial boundary $x_{j}= \pm \ell_{j}$.

The original problem is

$$
L\left(\partial_{t}, \partial_{x}\right) U=F
$$

with $F$ compactly supported in $R \subset \mathcal{D}$.

Bérenger's method introduces split dependent variables $\widetilde{U}:=\left(U^{1}, \ldots, U^{d}\right)$ with $U^{j}$ defined on $\mathcal{D} \backslash \overline{\mathcal{R}}$ and taking values in $\mathbb{C}^{N}$ for $1 \leq j \leq d$. Then $\widetilde{U}$ with values in $\mathbb{C}^{N d}$ is required to satisfy in the layers $\mathcal{D} \backslash(\overline{\mathcal{R}} \cup \mathcal{N})$,

$$
\left(\widetilde{L}\left(\partial_{t}, \partial_{x}\right) \widetilde{U}\right)_{j}:=\partial_{t} U^{j}+A_{j}(x) \partial_{j}\left(\sum_{\ell=1}^{d} U^{\ell}\right)+\sigma_{j}\left(x_{j}\right) U^{j}=0, \quad 1 \leq j \leq d
$$


Each absorption coefficient $\sigma_{j}\left(x_{j}\right) \geq 0$ depends on only one variable and vanishes in $\mathcal{R}$. There is in addition a $C^{k}$ valued $U_{B}$ defined on $\mathcal{R}$ and satisfying

$$
L\left(\partial_{t}, \partial_{x}\right) U_{B}=F \quad \text { on } \mathcal{R} .
$$

The partial differential equations (1.2) and (1.3) are supplemented by transmission conditions requiring that for each $k$ the function that is equal to $A_{k} \sum_{j} \widetilde{U}_{j}$ in $\mathcal{D} \backslash(\overline{\mathcal{R}} \cup \mathcal{N})$ and equal to $A_{k} U_{B}$ on $\mathcal{R}$ is continuous across the hyperplanes in $\mathcal{N}$ parallel to $\left\{x_{k}=0\right\}$. The exact form of the continuity depends on the existence proof. It is not made explicit here since it changes from case to case while the idea of the proof is not modified. In addition $\widetilde{U}$ must satisfy boundary conditions on $\partial \mathcal{D}$ that are constructed to be weakly reflective. It is only very recently that existence and uniqueness theorems have been proved for this transmission/boundary value problem with many corners. The corners are multihedral when $d \geq 3$.

The goal of Bérenger's strategy is that $U_{B}$ should be a good approximation to $U$ on $\mathcal{R}$.

Definition 1.1. The method is perfectly matched when Bérenger's transmission boundary value problem is at least weakly well posed and for $F$ supported in $t \geq 0$, the unique solution, also supported in $t \geq 0$, satisfies the perfection condition

$$
U_{B}=\left.U\right|_{\mathbb{R} \times \mathcal{R}}
$$

If the coefficients $A_{j}$ are constant on a neighborhood of the external layer $\mathcal{D} \backslash \mathcal{R}$, then the method is perfectly matched as soon as it is weakly well posed [16].

We give examples with variable coefficients at the layer that are not perfectly matched. The examples are from important applications where the Bérenger split system is well posed. This is the typical behavior to be expected when the coefficients are variable on a neighborhood of the interfaces.

Section 2 recalls the rigorous proof of perfection that works when the coefficients are constant on a neighborhood of the layer. The proof clearly fails when there are variable coefficients. This does not prove imperfection.

In Sections 3 and 4 infinitely accurate asymptotic expansions of reflected waves are constructed for variable coefficient problems. The phases satisfy variable coefficient nonlinear eikonal equations. Examples are constructed so that the phases are explicit. Since all the methods considered are cleverly designed, there are no reflections in the leading terms. Equivalently, a naive plane wave analysis would not reveal reflections. For Bérenger's method and the tuned layers the reflections are smaller by two powers of $\varepsilon$. This is a computation in perturbation theory beyond orders normally calculated.

The predictions from the asymptotic expansions are confirmed by numerical experiments in Section 5. Given the failure of perfection of Bérenger's method we compare the performance of that method and the so-called smart and tuned layers. In part this is done because the computation of the reflections for those methods is simpler than for Bérenger's method so is an excellent way to introduce the analysis. Numerical simulations reveal the reflections proved to exist by the asymptotic expansion.

Acknowledgment. Excellent suggestions from both referees, in particular T. Hagström, resulted in substantial improvements in this article. 


\section{Perfection and the change of variables method}

The proof of perfection in the case of constant coefficients from [16] is not bothered by variable coefficients so long as the coefficients are constant throughout the layer. We demonstrate in this article that variable coefficients in a neighborhood of the interface at the boundary of the layer can destroy perfection.

Recall the constant coefficient proof to see its failure when the coefficients vary at the interface.

Theorem 2.1. If $\mathcal{D}=\mathbb{R}^{d}$ and the Bérenger layer with absorptions vanishing in $\mathcal{R}$ defines an transmission problem that is at least weakly well posed, then the layer is perfectly matched provided the coefficients $A_{j}(x)$ are independent of $x$ on $\mathbb{R}^{d} \backslash \overline{\mathcal{R}}$.

Proof. Consider the Laplace transform in time of the split variables $\widetilde{U}$. The transform is

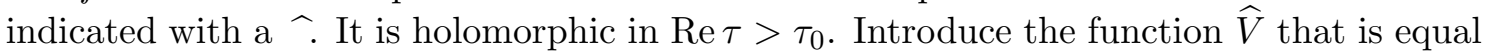
to $\mathcal{F}\left(\sum_{j} \widetilde{U}_{j}\right)$ on $\mathcal{D} \backslash \overline{\mathcal{R}}$ and equal to $\widehat{U}_{B}$ in $\mathcal{R}$.

The transform satisfies

$$
\widehat{U^{j}}+\left(\tau+\sigma_{j}\left(x_{j}\right)\right)^{-1} A_{j}(x) \partial_{j} \widehat{V}=0 \quad \text { on } \quad \mathbb{R}^{d} \backslash(\mathcal{R} \cup \mathcal{N}) .
$$

Multiply by $\tau$ and sum on $j$ to obtain the important equation that arises from the Laplace transform of the unsplit equation

$$
\tau \widehat{V}+\sum_{j} \frac{\tau}{\tau+\sigma_{j}\left(x_{j}\right)} A_{j}(x) \frac{\partial \widehat{V}}{\partial x_{j}}=0 \quad \text { on } \quad \mathbb{R}^{d} \backslash(\mathcal{R} \cup \mathcal{N}) .
$$

The Laplace transform of the equation satisfied by $U_{B}$ implies that this equation is also satisfied on $\mathcal{R}$. The transmission conditions across $\mathcal{N}$ imply that

$$
\tau \widehat{V}(x)+\sum_{j} \frac{\tau}{\tau+\sigma_{j}\left(x_{j}\right)} A_{j}(x) \frac{\partial \widehat{V}}{\partial x_{j}}(x)=\widehat{F}(x) \quad \text { on } \quad \mathbb{R}^{d} .
$$

The Laplace transform $\widehat{U}$ satisfies

$$
\tau \widehat{U}(x)+\sum_{j} A_{j}(x) \frac{\partial \widehat{U}}{\partial x_{j}}(x)=\widehat{F}(x) \quad \text { on } \quad \mathbb{R}^{d} .
$$

The key observation is that, at least when $\tau$ is real, (2.3) and (2.4) are conjugated one to the other by a change of variable leaving $\mathcal{R}$ and $\mathcal{N}$ fixed.

For fixed real $\tau>0$ define $d$ bilipschitzian homeomorphisms $X_{j}\left(x_{j}\right)$ of $\mathbb{R}$ to itself by

$$
\frac{d X_{j}\left(x_{j}\right)}{d x_{j}}=\frac{\tau+\sigma_{j}\left(x_{j}\right)}{\tau}, \quad X_{j}(0)=0
$$

Then,

$$
\frac{\partial}{\partial x_{j}}=\frac{d X_{j}}{d x_{j}} \frac{\partial}{\partial X_{j}}=\frac{\tau+\sigma_{j}\left(x_{j}\right)}{\tau} \frac{\partial}{\partial X_{j}}, \quad \frac{\tau}{\tau+\sigma_{j}\left(x_{j}\right)} \frac{\partial}{\partial x_{j}}=\frac{\partial}{\partial X_{j}} .
$$

Define $W(X)$ by $W(X)=\widehat{V}(x(X))$. Then

$$
\tau W(X)+\sum_{j} A_{j}(x(X)) \frac{\partial W}{\partial X_{j}}(X)=\widehat{F}(x(X)) .
$$


Since $x(X)=X$ on the support of $F$,

$$
\tau W(X)+\sum_{j} A_{j}(x(X)) \frac{\partial W}{\partial X_{j}}(X)=\widehat{F}(X) .
$$

The coefficents $A_{j}$ are constant on $\mathbb{R}^{d} \backslash \overline{\mathcal{R}}$ and $X \mapsto x(X)$ takes $\mathbb{R}^{d} \backslash \overline{\mathcal{R}}$ to itself. In addition, $x(X)=X$ on $\overline{\mathcal{R}}$. It follows that

$$
\tau W(X)+\sum_{j} A_{j}(X) \frac{\partial W}{\partial X_{j}}(X)=\widehat{F}(X) .
$$

The bilischizian map preserves $\mathcal{N}$ so $W$ satisfies the transmission conditions across $\mathcal{N}$. The function $X \mapsto W(X)$ solves exactly the same transmission problem (2.4) that defines the function $x \mapsto \widehat{U}(x)$.

The well posedness assumption implies that the transmission problem is uniquely solvable. It follows that

$$
X \mapsto W(X):=\widehat{V}(x(X)) \quad \text { and } \quad x \mapsto \widehat{U}(x)
$$

define the same functions on $\mathbb{R}^{d}$.

On $\mathcal{R}$ the bilipschitzian map is equal to the identity and $\widehat{V}=\widehat{U}_{B}$. Thus the identity just proved shows that on $\mathcal{R}, \widehat{U}_{B}=\widehat{U}$. Summarizing, for $x \in \mathcal{R}$ and for $\tau$ large and real,

$$
\widehat{U}_{B}(\tau, x)=\widehat{U}(\tau, x) \quad \text { on } \quad \mathcal{R} .
$$

The well posedness of the Bérenger problem and the hyperbolic problem imply that there is a $\lambda_{1}$ so that both sides of (2.8) are holomorphic in $\operatorname{Re} \tau>\lambda_{1}$. Since they are equal for large real $\tau$, unique continuation of holomorphic functions implies that they are equal in $\operatorname{Re} \tau>\lambda_{1}$. Uniqueness of the Laplace transform implies that

$$
U_{B}=U \quad \text { on } \mathcal{R} .
$$

This completes the proof of perfection.

Remark 2.1. T. Hagström, acting as a referee, observed that the above argument also proves perfection when $\mathcal{D}=\mathbb{R}^{d}$ and there is an absorbing layer only in $x_{j}$ (that is $\sigma_{k}=0$ for $k \neq j$ ) provided that in the layer, the coefficients are independent of $x_{j}$.

There are problems, for example with an isolated antenna for Maxwell's equations or radar in the exterior of an airplane where it is reasonable that the coefficients are constant outside $R$. For others, notably geophysical computations, the heterogeneity of the earth extends beyond $R$.

The failure of a proof of perfection does not prove imperfection. The following sections prove that Bérenger's method yield non trivial reflections.

\section{Analysis of reflection for a wave equation system}

To demonstrate imperfection we compute infinitely accurate high frequency asymptotic solutions of the transmission problem with variable coefficients at the interface between the unsplit region $x_{1}<0$ and the layer in $x_{1}>0$. The computation is performed on a 
$2 \times 2$ system that is as simple as possible. See [16] for similar computations for Maxwell's equations in $\mathbb{R}^{3}$ with constant coefficients.

The analysis of smart layers, which add a damping to the equation, is done in Section 3. The Bérenger's method splitting the equations as in (1.2) is analysed in Section 4. The interest of the smart layer is that it is dissipative for the natural $L^{2}$ norm. Bérenger's method often destroys strong hyperbolicity, for example in case of Maxwell's equations.

\subsection{Preliminaries}

Consider the wave equation with $0<c(\cdot) \in C^{\infty}(\mathbb{R})$,

$$
\begin{aligned}
& L_{1}(x, \partial) u:=\partial_{t} u+A_{1} \partial_{1} u+A_{2} \partial_{2} u=0 \quad \text { in } \mathbb{R}^{1+2}, \\
& A_{1}=c\left(x_{1}\right)\left(\begin{array}{ll}
0 & 1 \\
1 & 0
\end{array}\right), \quad A_{2}=c\left(x_{1}\right)\left(\begin{array}{cc}
1 & 0 \\
0 & -1
\end{array}\right) .
\end{aligned}
$$

Define

$$
L(x, \partial) u:=L_{1}(x, \partial) u+\sigma B u=0, \quad x_{1}>0 .
$$

$0<\sigma \in \mathbb{R}$. The matrix $B$ is a symmetric non negative matrix, for example the identity, or the projection on the positive eigenspace $E_{+}=\mathbb{C} \Phi_{+}=\mathbb{C}(1,1)$ of $A_{1}$, that is

$$
B_{+}=\frac{1}{2}\left(\begin{array}{ll}
1 & 1 \\
1 & 1
\end{array}\right)
$$

This choice is called the smart layer in [16].

\subsection{Incident asymptotic solution in $x_{1}<0$.}

The following computations are justified by the analysis in Chapter 5 of [26]. The ansatz for solutions of $L u \sim 0$ is

$$
u^{\varepsilon} \sim e^{i S / \varepsilon} \sum_{j=0}^{+\infty} \varepsilon^{j} a_{j}(t, x), \quad \text { with the phase } S(t, x)=t+\varphi\left(x_{1}\right)+k x_{2} .
$$

The candidate $u^{\varepsilon}$ oscillates in time with the frequency $1 / 2 \pi \varepsilon>>1$. The real number $k$ is fixed. The differential of the phase is then given by $d S=\left(1, \varphi^{\prime}, k\right)$.

Theorem 3.1. (i) If the real valued function $\varphi \in C^{\infty}$ with $\varphi^{\prime} \neq 0$ satisfies the eikonal equation

$$
c^{2}\left(x_{1}\right)\left(\varphi^{\prime 2}\left(x_{1}\right)+k^{2}\right)=1,
$$

(ii) and the coefficients $a_{j} \in C^{\infty}$ satisfy the recursion relation

$$
\begin{aligned}
& a_{0}(t, x) \in \operatorname{Ker} L_{1}(x, d S), \\
& \forall j \geq 0, \quad i L_{1}(x, d S) a_{j+1}(t, x)+L(x, \partial) a_{j}(t, x)=0,
\end{aligned}
$$

then (3.4) is an infinitely accurate approximate solution of $L u=0$. We write $L u^{\varepsilon}=$ $O\left(\varepsilon^{\infty}\right)$. This means that for all $N$ and compact $K,\left\|L\left(\partial_{t}, \partial_{x}\right) u^{\varepsilon}\right\|_{L^{\infty}(K)}=O\left(\varepsilon^{N}\right)$. 
(iii) If $g_{j}=\Pi g_{j} \in C_{0}^{\infty}\left(\mathbb{R}^{2}\right)$ ( $\Pi$ is the projection operator on $\operatorname{Ker} L_{1}(x, d S)$ ) are supported in a fixed compact $K$, then there is one and only one family of $a_{j}$ satisfying (3.5) together with the initial conditions, $\Pi a_{j}(0, \cdot)=g_{j}$ and the polarization $\Pi a_{0}=a_{0}$. They have support in the tube of rays with feet in $K$ and speed of propagation given by the group velocity

$$
\mathbf{v}=-c^{2}\left(\varphi^{\prime}, k\right) \text {. }
$$

(iv) Let $\Phi$ be the generator of $\operatorname{Ker} L_{1}(x, d S)$ defined in (3.13). The principal term $a_{0}(t, x)=$ $\alpha_{0} \Phi$ is a solution of the transport equation

$$
\partial_{t} \alpha_{0}+\boldsymbol{v} \cdot \nabla \alpha_{0}+(d+\gamma \sigma) \alpha_{0}=0
$$

$\gamma$ and $d$ are defined by

$$
\Pi B \Phi=\gamma \Phi, \quad \Pi A_{1} \Phi^{\prime}=d \Phi, \quad d=\frac{k c^{\prime}(2 c k-1)}{2 \varphi^{\prime}} .
$$

Proof. The computation is not simple to carry out, so we present the main details. Inject the ansatz in $L u^{\varepsilon}=\left(L_{1}+\sigma B\right) u^{\varepsilon} \sim 0$ to find

$$
L\left(e^{i S / \varepsilon} \sum \varepsilon^{j} a_{j}\right) \sim e^{i S / \varepsilon}\left(\frac{i}{\varepsilon} L_{1}(x, d S) \sum_{j \geq 0} \varepsilon^{j} a_{j}+\sum_{j \geq 0} \varepsilon^{j} L(x, \partial) a_{j}\right)=0,
$$

with

$$
L_{1}(x, d S)=I+\varphi^{\prime} A_{1}+k A_{2}=\left(\begin{array}{cc}
1+k c & c \varphi^{\prime} \\
c \varphi^{\prime} & 1-k c
\end{array}\right) .
$$

The term of order -1 of the expansion above gives

$$
L_{1}(x, d S) a_{0}=0 .
$$

In order to have a nontrivial solution $a_{0}$ this requires the eikonal equation,

$$
\operatorname{det} L_{1}(x, d S)=0, \quad \text { equivalently, } \quad c^{2}\left(\varphi^{\prime 2}+k^{2}\right)=1 .
$$

We suppose that $k$ is such that $k c\left(x_{1}\right)<1$. Define $\varphi_{+}$by

$$
\varphi_{+}^{\prime}\left(x_{1}\right)=\sqrt{\frac{1}{c^{2}\left(x_{1}\right)}-k^{2}}, \quad \varphi_{+}(0)=0 .
$$

There are two solutions to the eikonal equation, according to the sign of $\varphi^{\prime}$. The group velocity shows that solution with positive (resp. negative) $\varphi^{\prime}$ is a leftward propagating wave (resp. rightward). The kernel of $L_{1}(x, d S)$ is one-dimensional, spanned by

$$
\Phi=\left(-c \varphi^{\prime}, 1+k c\right)
$$

Thanks to the eikonal equation, its norm is given by $\|\Phi\|^{2}=2(1+k c)$. The image, Im $L_{1}(x, d S)$, is one dimensional, with basis $\Psi:=\left(1+k c, c \varphi^{\prime}\right) .(\Phi, \Psi)$ are an orthogonal basis for $\mathbb{R}^{2}$. The orthogonal projection on the kernel is denoted by $\Pi(x, d S)$ :

$$
\Pi:=\frac{\Phi \Phi^{*}}{\Phi^{*} \Phi}, \quad \text { equivalently }, \quad \Pi w=\frac{(\Phi, w)}{\|\Phi\|^{2}} \Phi .
$$


By (3.10), $a_{0}$ is polarized, i.e. takes values in the kernel of $L_{1}(x, d S)$,

$$
a_{0}=\Pi a_{0}
$$

These choices of the phase and $a_{0}$ guarantee that the leading term in the first sum of (3.2) vanishes so the first sum is for $j \geq 1$ while the second is for $j \geq 0$. Setting the term of order $\varepsilon^{j}$ equal to zero for all $j \geq 0$ requires that the next terms in the expansion satisfy

$$
\forall j \geq 0, \quad i L_{1}(x, d S) a_{j+1}(t, x)+L(x, \partial) a_{j}(t, x)=0 .
$$

Denote by $Q(x, d S)$ the partial inverse of $L_{1}(x, d S)$ defined by

$$
Q(x, d S) \Pi(x, d S)=0, \quad Q(x, d S) L_{1}(x, d S)=I-\Pi(x, d S) .
$$

Multiplying (3.16) on the left, first by the orthogonal projection $\Pi(x, d S)$, and second by $Q(x, d S)$, yield the pair of equations equivalent to the $j$-equation,

$$
\begin{array}{r}
\Pi(x, d S) L(x, \partial) a_{j}=0, \\
i(I-\Pi(x, d S)) a_{j+1}+Q(x, d S) L(x, \partial) a_{j}=0 .
\end{array}
$$

These equations are the starting point for the recursive computation of the $a_{j}$. Equation (3.17a) for $j=0$ gives a transport equation on $a_{0}=\Pi a_{0}$ :

$$
\Pi(x, d S) L(x, \partial) a_{0}(t, x):=\Pi(x, d S)\left(\partial_{t} a_{0}+A_{1} \partial_{1} a_{0}+A_{2} \partial_{2} a_{0}+\sigma B a_{0}\right)=0 .
$$

Define $\alpha_{0}$ by $a_{0}=\alpha_{0} \Phi$. Since $\Phi$ only depends on $x_{1}$ one has

$$
\begin{gathered}
\partial_{1}\left(\alpha_{0} \Phi\right)=\partial_{1} \alpha_{0} \Phi+\alpha_{0} \Phi^{\prime}, \quad \partial_{2}\left(\alpha_{0} \Phi\right)=\partial_{2} \alpha_{0} \Phi, \quad \text { and } \quad \partial_{t}\left(\alpha_{0} \Phi\right)=\partial_{t} \alpha_{0} \Phi, \quad \text { so } \\
\left(\partial_{t} \alpha_{0}+\partial_{1} \alpha_{0} \Pi A_{1}+\partial_{2} \alpha_{0} \Pi A_{2}\right) \Phi+\alpha_{0}\left(\sigma \Pi B \Phi+\Pi A_{1} \Phi^{\prime}\right)=0 .
\end{gathered}
$$

The $\Pi A_{j} \Pi$ are related to the group velocity by (see [26])

$$
\Pi A_{j} \Pi=v_{j} \Pi, \quad v_{j}=\frac{\left(A_{j} \Phi, \Phi\right)}{\|\Phi\|^{2}} .
$$

This gives the formula for the group velocity in (3.6). Defining $d$ and $\gamma$ by (3.8) yields (3.7). In $x_{1}<0$, the transport equation together with initial value $g_{0}$, determines $\Pi a_{0}$. The process iterates until all coefficients are determined as follows. Given $a_{j-1},(I-\Pi) a_{j}$ is explicitly given by

$$
(I-\Pi) a_{j}=i Q(x, d S) L(x, \partial) a_{j-1}
$$

Then (3.17a) is split as

$$
\Pi(x, d S) L(x, \partial) \Pi a_{j}=-\Pi(x, d S) L(x, \partial)(I-\Pi) a_{j},
$$

and gives a transport equation on $\alpha_{j}$ defined by $\Pi a_{j}=\alpha_{j} \Phi$ as before. This transport equation, with initial data given by $g_{j}$, uniquely determines $a_{j}$. For the precise formulas, note that $\Pi(x, d S)$ is given by

$$
\Pi=\frac{1}{2}\left(\begin{array}{cc}
1-k c & -c \varphi^{\prime} \\
-c \varphi^{\prime} & 1+k c
\end{array}\right)
$$


Therefore

$$
I-\Pi=\frac{1}{2} L_{1}(x, d S), \quad L_{1}(x, d S)=2(I-\Pi)+0 \Pi .
$$

To calculate $d$, we must calculate $\Phi^{\prime}=\left(-\left(c \varphi^{\prime}\right)^{\prime}, k c^{\prime}\right)$. Differentiate the eikonal equation written as $\left(c \varphi^{\prime}\right)^{2}+k^{2} c^{2}=1$ to find

$$
2\left(c \varphi^{\prime}\right)\left(c \varphi^{\prime}\right)^{\prime}+2 k^{2} c c^{\prime}=0, \quad \text { which gives } \quad-\varphi^{\prime} \Phi_{1}^{\prime}+k \Phi_{2}^{\prime}=0 .
$$

Therefore

$\Phi^{\prime}=\frac{\Phi_{2}^{\prime}}{\varphi^{\prime}}\left(\begin{array}{c}k \\ \varphi^{\prime}\end{array}\right)=\frac{k c^{\prime}}{\varphi^{\prime}}\left(\begin{array}{c}k \\ \varphi^{\prime}\end{array}\right), \quad A_{1} \Phi^{\prime}=\frac{k c c^{\prime}}{\varphi^{\prime}}\left(\begin{array}{c}\varphi^{\prime} \\ k\end{array}\right), \quad \Pi A_{1} \Phi^{\prime}=\frac{k c c^{\prime}}{2 \varphi^{\prime}(1+k c)}\left(\begin{array}{c}\varphi^{\prime} \\ k\end{array}\right) \cdot\left(\begin{array}{c}-c \varphi^{\prime} \\ 1+k c\end{array}\right) \Phi$.

that yield the second part of formula (3.8), and (3.7). The transport at step $j$ follows the same dynamics, with a right-hand side:

$$
\left(\partial_{t} \alpha_{j}+\boldsymbol{v} \cdot \nabla \alpha_{j}+(d+\gamma \sigma) \alpha_{j}\right) \Phi=-\Pi(x, d S) L(x, \partial)(I-\Pi) a_{j} .
$$

For future use compute $Q(x, d S)$. By the Cayley-Hamilton theorem, we have $\left(L_{1}(x, d S)\right)^{2}=$ $2 L_{1}(x, d S)$, therefore using (3.22), we see that $Q$ is given by

$$
Q(x, d S)=\frac{1}{2}(I-\Pi(x, d S))=\frac{1}{4} L_{1}(x, d S) .
$$

\subsection{Reflection for the wave equation with layer}

The analysis is made in $\mathbb{R}^{2}$. The velocity $c$ is assumed to be smooth. The coefficient $\sigma$ in contrast may be discontinuous at $x_{1}=0$. Solving the Cauchy problem in $\mathbb{R}^{2}$ with a discontinuous coefficient $\sigma$ is equivalent to solving the problem in each half-space, with transmission condition $\left[A_{1} u\right]_{\Gamma}=0$. Since $A_{1}$ is invertible, this is equivalent to $[u]_{\Gamma}=0$.

Next analyse the reflection and transmission of a high frequency wave in $x_{1} \leq 0$ when it arrives at the boundary $x_{1}=0$. The input is a solution with phase $S^{\mathrm{I}}(t, x):=$ $t-\varphi_{+}\left(x_{1}\right)+k x_{2}$,

$$
u^{\varepsilon}:=e^{i S^{\mathrm{I}}(t, x) / \varepsilon} a^{\mathrm{I}}(t, x, \varepsilon), \quad a^{\mathrm{I}}(t, x, \varepsilon) \sim \sum_{j=0}^{\infty} \varepsilon^{j} a_{j}^{\mathrm{I}}(t, x), \quad L_{1}\left(\partial_{t}, \partial_{x}\right) u^{\varepsilon}=O\left(\varepsilon^{\infty}\right) .
$$

Suppose that the amplitudes $a_{j}^{\mathrm{I}}$ are supported in a tube $T \subset \mathbb{R}^{1+2}$ of rays with temporal cross sections $T \cap\{t=0\}$ compact in $\left\{x_{1}<0\right\}$.

Using Theorem 3.1, we construct a transmitted wave with the same phase and a reflected wave, supported in $x_{1} \leq 0$, with phase $S^{\mathrm{R}}(t, x):=t+\varphi_{+}\left(x_{1}\right)+k x_{2}$. The reflected wave $v^{\varepsilon}$ is

$$
v^{\varepsilon}=e^{i S^{\mathrm{R}}(t, x) / \varepsilon} a^{\mathrm{R}}(t, x, \varepsilon), \quad a^{\mathrm{R}}(t, x, \varepsilon) \sim \sum_{j=0}^{\infty} \varepsilon^{j} a_{j}^{\mathrm{R}}(t, x), \quad L_{1}\left(\partial_{t}, \partial_{x}\right) v^{\varepsilon}=O\left(\varepsilon^{\infty}\right) .
$$

The transmitted wave, supported in $x_{1} \geq 0$, is

$$
w^{\varepsilon}:=e^{i S^{\mathrm{I}}(t, x) / \varepsilon} a^{\mathrm{T}}(t, x, \varepsilon), \quad a^{\mathrm{T}}(t, x, \varepsilon) \sim \sum_{j=0}^{\infty} \varepsilon^{j} a_{j}^{\mathrm{T}}(t, x), \quad L\left(\partial_{t}, \partial_{x}\right) w^{\varepsilon}=O\left(\varepsilon^{\infty}\right) .
$$

They both have vanishing initial values. We first show that there are uniquely determined reflected and transmitted waves. Then we compute exactly the leading terms in their asymptotic expansions. 


\section{Theorem 3.2.}

(i) Given the incoming amplitudes $a_{j}^{I}$ there are uniquely determined amplitudes $a_{j}^{T}$ and $a_{j}^{R}$ so that $u^{\varepsilon}, v^{\varepsilon}$ and $w^{\varepsilon}$ are infinitely accurate solutions of the differential equations and the transmission condition is also satisfied to infinite order,

$$
\forall\left(t, x_{2}\right) \in \mathbb{R} \times \mathbb{R}, \quad A_{1}\left(u^{\varepsilon}+v^{\varepsilon}\right)\left(t, 0_{-}, x_{2}\right)=A_{1} w^{\varepsilon}\left(t, 0_{+}, x_{2}\right)+O\left(\varepsilon^{\infty}\right) .
$$

(ii) If $\sigma\left(x_{1}\right)=\sigma \mathbf{1}_{x_{1}>0}$, the coefficient $a_{0}^{R}$ vanishes identically, and the reflection coefficient is

$$
R_{1}(k)=i \sigma \frac{(1+k c(0)) b_{22}-(1-k c(0)) b_{11}}{4 c^{2}(0) \varphi_{+}^{\prime 2}(0)} .
$$

That is, noting $a_{0}^{I}(t, x)=\alpha_{0}^{I}(t, x) \Phi^{I} \in \operatorname{Ker} L_{1}\left(0, d S^{I}\right)$, then

$$
\forall\left(t, x_{2}\right) \in \mathbb{R} \times \mathbb{R}, \quad a_{1}^{R}\left(t, 0_{-}, x_{2}\right)=R_{1}(k) \alpha_{0}^{I}\left(t, 0_{-}, x_{2}\right) \Phi^{R} .
$$

(iii) For a matrix $B$, the reflection coefficient $R_{1}(k)$ vanishes if and only if the diagonal part of $B$ is a scalar multiple of the matrix

$$
B(k)=\left(\begin{array}{cc}
1+k c & 0 \\
0 & 1-k c
\end{array}\right) .
$$

In that case the first nonzero reflection coefficient then occurs at least at order 2 , so

$$
\forall\left(t, x_{2}\right) \in \mathbb{R} \times \mathbb{R}, \quad a_{2}^{R}\left(t, 0_{-}, x_{2}\right)=R_{2}\left(k, \partial_{2}\right) \alpha_{0}^{I}\left(t, 0_{-}, x_{2}\right) \Phi^{R} .
$$

These are called tuned layers. For the tuned layer, the reflection operator is

$$
R_{2}\left(k, \partial_{2}\right)=\frac{\sigma}{4 c^{2}(0) \varphi_{+}^{\prime 3}(0)}\left(k c^{\prime}(0)+2 c(0) \varphi_{+}^{\prime}(0) \partial_{2}\right) .
$$

\section{Remark 3.1.}

1. The reflection coefficient does not depend on the off-diagonal entries of $B$.

2. The reflection coefficient vanishes at normal incidence if and only if $b_{11}=b_{22}$.

3. The most common matrices $B$ are $B \equiv I$ and $B \equiv \Pi_{+}$defined in (3.3). In these cases

$$
R_{1}(k)=\frac{i \delta \sigma k}{4\left(c \varphi^{\prime 2}\right)(0)}, \quad \delta=\left\{\begin{array}{lll}
1 & \text { if } B \equiv \Pi_{+} & (a) \\
2 & \text { if } B \equiv I & (b)
\end{array}\right.
$$

Proof. ( $i$ ) The formulas in Theorem 3.1 and its proof apply to the incident and reflected waves in $x_{1} \leq 0$ with $B \equiv 0$, and to the transmitted wave in $x_{1} \geq 0$. The phases are $\varphi^{\mathrm{I}, \mathrm{T}}=-\varphi_{+}=-\varphi^{\mathrm{R}}$. The corresponding kernel, projector, and partial inverse of $L_{1}(x, d S)$ are indexed by $I, R$, or $T$. They are the same for the incident and transmitted waves. To determine the coefficients $a_{j}^{\mathrm{I}, \mathrm{R}, \mathrm{T}}$ requires the transmission condition $A_{1}\left(u^{\varepsilon}+v^{\varepsilon}\right)=A_{1} w^{\varepsilon}$ on $x_{1}=0$. This is equivalent to

$$
a_{j}^{\mathrm{I}}+a_{j}^{\mathrm{R}}=a_{j}^{\mathrm{T}} \quad \text { on } \quad x_{1}=0, \quad j=0,1, \ldots
$$


The polarized parts are written with obvious notations $\Pi^{\mathrm{I}, \mathrm{I}, \mathrm{R}} a_{j}^{\mathrm{I}, \mathrm{T}, \mathrm{R}}=\alpha_{j}^{\mathrm{I}, \mathrm{T}, \mathrm{R}} \Phi^{\mathrm{I}, \mathrm{I}, \mathrm{R}}$. The coefficients $\alpha_{j}^{\mathrm{I}, \mathrm{T}, \mathrm{R}}$ are solutions of transport equations in the half spaces. They are determined by the transmission condition. Details follow for $j=0,1$. For the tuned layer, we treat also $j=2$.

(ii) By (3.15) the coefficients at order 0 are polarized, $\Pi^{\mathrm{I}, \mathrm{R}, \mathrm{T}} a_{0}^{\mathrm{I}, \mathrm{R}, \mathrm{T}}=a_{0}^{\mathrm{I}, \mathrm{R}, \mathrm{T}}$. The transmission condition at order 0 becomes

$$
\Pi^{\mathrm{I}} a_{0}^{\mathrm{I}}+\Pi^{\mathrm{R}} a_{0}^{\mathrm{R}}=\Pi^{\mathrm{T}} a_{0}^{\mathrm{T}} \quad \text { on } x_{1}=0
$$

equivalently,

$$
\alpha_{0}^{\mathrm{R}} \Phi^{\mathrm{R}}=\left[\alpha_{0}\right] \Phi^{\mathrm{I}} \text { on } x_{1}=0,
$$

where $[\alpha]$ denotes the jump between the transmitted and incident part at $x_{1}=0$, that is $[\alpha]\left(t, x_{2}\right)=\alpha^{\mathrm{T}}\left(t, 0_{+}, x_{2}\right)-\alpha^{\mathrm{I}}\left(t, 0_{-}, x_{2}\right)$. Since $\Phi^{\mathrm{I}}=\left(-c\left(\varphi^{\mathrm{I}}\right)^{\prime}, 1+k c\right)$ and $\Phi^{\mathrm{R}}=\left(-c\left(\varphi^{\mathrm{R}}\right)^{\prime}, 1+\right.$ $k c)=\left(c\left(\varphi^{\mathrm{I}}\right)^{\prime}, 1+k c\right)$, these two vectors are transverse, so

$$
\alpha_{0}^{\mathrm{R}}=0 \quad \text { and } \quad \alpha_{0}^{\mathrm{I}}=\alpha_{0}^{\mathrm{T}} \quad \text { on } x_{1}=0 .
$$

The transport equation (3.7) for the incident, reflected, and transmitted waves becomes with $v_{1}^{\mathrm{I}}=v_{1}^{\mathrm{T}}=-c^{2} \varphi^{\mathrm{I}}=c^{2} \varphi_{+}^{\prime}=-v_{1}^{\mathrm{R}}, v_{2}=-k c^{2}$,

$$
\begin{array}{ll}
\partial_{t} \alpha_{0}^{\mathrm{I}}+v_{1}^{\mathrm{I}} \partial_{1} \alpha_{0}^{\mathrm{I}}+v_{2} \partial_{2} \alpha_{0}^{\mathrm{I}}+d^{\mathrm{I}} \alpha_{0}^{\mathrm{I}}=0, & x_{1}<0, \\
\partial_{t} \alpha_{0}^{\mathrm{T}}+v_{1}^{\mathrm{I}} \partial_{1} \alpha_{0}^{\mathrm{T}}+v_{2} \partial_{2} \alpha_{0}^{\mathrm{T}}+\left(d^{\mathrm{I}}+\gamma \sigma\right) \alpha_{0}^{\mathrm{T}}=0, & x_{1}>0, \\
\partial_{t} \alpha_{0}^{\mathrm{R}}+v_{1}^{\mathrm{R}} \partial_{1} \alpha_{0}^{\mathrm{R}}+v_{2} \partial_{2} \alpha_{0}^{\mathrm{R}}+d^{\mathrm{R}} \alpha_{0}^{\mathrm{R}}=0, & x_{1}<0 .
\end{array}
$$

Since $a_{0}^{\mathrm{R}}$ has zero initial value and boundary data at $x_{1}=0$, it vanishes identically in $\mathbb{R}_{-}^{2}$. The reflection is at least first order. Next determine inductively the correctors. By (3.19), $\left(I-\Pi^{\mathrm{R}}\right) a_{1}^{\mathrm{R}} \equiv 0$ in $\mathbb{R}_{-}^{2}$ and $a_{1}^{\mathrm{R}}$ is polarized. Therefore, splitting the transmission condition (3.34) for $j=1$, one finds on the interface

$$
\begin{gathered}
\Pi^{\mathrm{I}} a_{1}^{\mathrm{I}}-\Pi^{\mathrm{T}} a_{1}^{\mathrm{T}}+\Pi^{\mathrm{R}} a_{1}^{\mathrm{R}}=-(I-\Pi) a_{1}^{\mathrm{I}}+(I-\Pi) a_{1}^{\mathrm{T}}, \quad \text { so } \\
-\left[\alpha_{1}\right] \Phi^{\mathrm{I}}+\alpha_{1}^{\mathrm{R}} \Phi^{\mathrm{R}}=\left[(I-\Pi) a_{1}\right] \text { on } x_{1}=0 .
\end{gathered}
$$

For the incident and transmitted phases and amplitudes, the superscripts, $I, T$, are omitted when no confusion is to be feared. Next identify the righthand side of (3.36). From (3.19)

$$
(I-\Pi) a_{1}=i Q\left(\partial_{t}+A_{1} \partial_{1}+A_{2} \partial_{2}+\sigma B\right) a_{0}
$$

with $\sigma \equiv 0$ for the incident wave. Insert $a_{0}=\prod a_{0}=\alpha_{0}(t, x) \Phi$. By definition of $Q$, $Q a_{0}=0$, and therefore $Q \partial_{t} a_{0}=\left(\partial_{t} \alpha_{0}\right) Q \Phi=0$. Furthermore, $\Phi$ depends only on $x_{1}$. So,

$$
(I-\Pi) a_{1}=i Q\left(\left(\partial_{1} \alpha_{0} A_{1}+\partial_{2} \alpha_{0} A_{2}+\alpha_{0} \sigma B\right) \Phi+\alpha_{0} A_{1} \Phi^{\prime}\right) .
$$

Introduce the transverse part of the transport operator

$$
\mathcal{T}:=\partial_{t}+v_{2} \partial_{2}+d
$$

to rewrite the transport equations for the incident and transmitted waves in (3.35) as

$$
v_{1} \partial_{1} \alpha_{0}+\mathcal{T} \alpha_{0}+\gamma \sigma \alpha_{0}=0, \quad \sigma=0 \text { for } x_{1}<0 .
$$


Replace $\partial_{1} \alpha_{0}$ in (3.37) to obtain

$$
(I-\Pi) a_{1}=i Q\left(\left(-\frac{1}{v_{1}^{\mathrm{I}}}(\mathcal{T}+\gamma \sigma) \alpha_{0} A_{1}+\partial_{2} \alpha_{0} A_{2}+\alpha_{0} \sigma B\right) \Phi+\alpha_{0} A_{1} \Phi^{\prime}\right),
$$

or, reordering the terms,

$$
(I-\Pi) a_{1}=i Q\left(\left(\partial_{2} \alpha_{0} A_{2}-\frac{1}{v_{1}} \mathcal{T} \alpha_{0} A_{1}\right) \Phi+\alpha_{0} A_{1} \Phi^{\prime}+\alpha_{0} \sigma\left(B-\frac{\gamma}{v_{1}} A_{1}\right) \Phi\right) .
$$

The evaluation of the important term with $\sigma$ is the object of the following lemma.

Lemma 3.3. For any symmetric matrix $B$, define the matrix $B_{1}=B-\frac{\gamma}{v_{1}} A_{1}$, with $\gamma$ defined by $\Pi B \Phi=\gamma \Phi$. Then

$$
\Pi B_{1} \Phi=0, \quad Q B_{1} \Phi=\frac{b_{22}(1+k c)-b_{11}(1-k c)}{4 c \varphi^{\prime}} \Psi .
$$

Proof of Lemma. First it is easy to see that

$$
\Pi\left(B-\frac{\gamma}{v_{1}} A_{1}\right) \Phi=\gamma \Phi-\frac{\gamma}{v_{1}} \Pi A_{1} \Phi=0 .
$$

Therefore $\left(B-\frac{\gamma}{v_{1}} A_{1}\right) \Phi$ is parallel to $\Psi=L_{1} e_{1}$, and since $Q=\frac{1}{2}(I-\Pi)$,

$$
Q\left(B-\frac{\gamma}{v_{1}} A_{1}\right) \Phi=\frac{1}{2}\left(B-\frac{\gamma}{v_{1}} A_{1}\right) \Phi
$$

Suppose now that (using the orthogonal basis $(\Phi, \Psi)$ with $\|\Phi\|^{2}=\|\Psi\|^{2}$ )

$$
B \Phi=\gamma \Phi+\eta \Psi, \quad \gamma=\frac{(B \Phi, \Phi)}{\|\Phi\|^{2}}, \quad \eta=\frac{(B \Phi, \Psi)}{\|\Phi\|^{2}}
$$

compute $A_{1} \Phi=v_{1} \Phi-v_{2} \Psi$, and therefore

$$
\left(B-\frac{\gamma}{v_{1}} A_{1}\right) \Phi=\left(\eta+\gamma \frac{v_{2}}{v_{1}}\right) \Psi .
$$

Then

$$
\eta+\gamma \frac{v_{2}}{v_{1}}=\eta+\gamma \frac{k}{\varphi^{\prime}}=\frac{1}{\|\Phi\|^{2}}\left(B \Phi, \Psi+\frac{k}{\varphi^{\prime}} \Phi\right) .
$$

Compute

$$
\Psi+\frac{k}{\varphi^{\prime}} \Phi=\frac{1}{c \varphi^{\prime}}\left(\begin{array}{c}
c \varphi^{\prime} \\
1+k c
\end{array}\right), \quad \text { therefore } \eta+\gamma \frac{v_{2}}{v_{1}}=\frac{b_{22}(1+k c)-b_{11}(1-k c)}{2 c \varphi^{\prime}}
$$

Use this in (3.43) to get $\left(B-\frac{\gamma}{v_{1}} A_{1}\right) \Phi$, and then into (3.43) to get the third equality in (3.41).

End of Proof of Theorem. Since $c$, and hence $v_{1}$, is smooth across the interface $x_{1}=0$, the righthand side of $(I-\Pi) a_{1}$ is composed of two parts: one is continuous across the interface, the other one having $\sigma$ as a factor, jumps. For the jump, we find

$$
\left[(I-\Pi) a_{1}\right]=i \sigma \alpha_{0} Q B_{1} \Phi \quad \text { at } x_{1}=0 .
$$


Insert (3.41) in (3.44) to find

$$
\left[\left(I-\Pi^{\mathrm{I}}\right) a_{1}\right]=i \sigma \alpha_{0}^{\mathrm{I}} \frac{b_{22}(1+k c)-b_{11}(1-k c)}{4 c \varphi^{\mathrm{I}^{\prime}}} \Psi^{\mathrm{I}} .
$$

Replace in the transmission condition (3.36), to find

$$
-\left[\alpha_{1}\right] \Phi^{\mathrm{I}}+\alpha_{1}^{\mathrm{R}} \Phi^{\mathrm{R}}=i \sigma \alpha_{0}^{\mathrm{I}} \frac{b_{22}(1+k c)-b_{11}(1-k c)}{4 c \varphi^{\mathrm{I}}} \Psi^{\mathrm{I}} .
$$

Take the scalar product with $\Psi^{\mathrm{I}}$ in $(3.45)$ to obtain, since $\Phi^{\mathrm{I}}$ and $\Psi^{\mathrm{I}}$ are orthogonal,

$$
\alpha_{1}^{\mathrm{R}}=i \sigma \alpha_{0}^{\mathrm{I}} \frac{b_{22}(1+k c)-b_{11}(1-k c)}{4 c \varphi^{\mathrm{I}}} \frac{\left(\Psi^{\mathrm{I}}, \Psi^{\mathrm{I}}\right)}{\left(\Psi^{\mathrm{I}}, \Phi^{\mathrm{R}}\right)} .
$$

Compute

$$
\frac{\left(\Psi^{\mathrm{I}}, \Psi^{\mathrm{I}}\right)}{\left(\Psi^{\mathrm{I}}, \Phi^{\mathrm{R}}\right)}=\frac{1}{c \varphi^{\mathrm{I}}}
$$

to obtain formula (3.29) in Theorem 3.2.

(iii) Furthermore, no reflection occurs at this order if and only if $b_{22}(1+k c)-b_{11}(1-$ $k c)=0$, that is $\left[(I-\Pi) a_{1}\right]=0$ and $\left[\alpha_{1}\right]=\alpha_{1}^{\mathrm{R}}=0$. This proves the first part of (iii) in Theorem 3.2.

Computation of the order two reflection. Since the extra diagonal coefficients do not intervene, we suppose from now on that $B=B(k)$ from (3.31). Then

$$
\gamma=c^{2} \varphi^{\prime 2}, \quad B_{1}:=B-\frac{\gamma}{v_{1}} A_{1}=L_{1}\left(x, d S^{\mathrm{I}}\right)
$$

Next compute the transport equation for $\alpha_{1}^{\mathrm{R}}$. Since $\left(I-\Pi^{\mathrm{R}}\right) \alpha_{1}^{\mathrm{R}} \equiv 0$ in $\mathbb{R}_{-}^{d},(3.35 \mathrm{c})$ is valid for $\alpha_{1}^{\mathrm{R}}$,

$$
\partial_{t} \alpha_{1}^{\mathrm{R}}+\boldsymbol{v}^{\mathrm{R}} \cdot \nabla \alpha_{1}^{\mathrm{R}}+d^{\mathrm{R}} \alpha_{1}^{\mathrm{R}}=0 .
$$

Again, $\alpha_{1}^{\mathrm{R}}$ being solution of the leftward transport equation with zero boundary and initial data, vanishes identically in $\mathbb{R}_{-}^{2}$. The information obtained so far is

$$
a_{1}^{\mathrm{R}}=\Pi^{\mathrm{R}} a_{1}^{\mathrm{R}} \equiv 0 \text { in } \mathbb{R}_{-}^{2}, \quad\left[a_{1}\right]=0 \text { on } x_{1}=0 .
$$

To calculate the next term in the expansion of the reflected wave, start with the transmission condition $a_{2}^{\mathrm{I}}+a_{2}^{\mathrm{R}}=a_{2}^{\mathrm{T}}$ on $x_{1}=0$. Splitting the amplitudes into $\Pi$ and $I-\Pi$ parts yields

$$
\Pi^{\mathrm{I}} a_{2}^{\mathrm{I}}+\Pi^{\mathrm{R}} a_{2}^{\mathrm{R}}+\left(I-\Pi^{\mathrm{I}}\right) a_{2}^{\mathrm{I}}+\left(I-\Pi^{\mathrm{R}}\right) a_{2}^{\mathrm{R}}=\Pi^{\mathrm{I}} a_{2}^{\mathrm{T}}+\left(I-\Pi^{\mathrm{I}}\right) a_{2}^{\mathrm{T}} \quad \text { on } x_{1}=0,
$$

rewritten as $\Pi^{\mathrm{R}} a_{2}^{\mathrm{R}}-\left[\Pi^{\mathrm{I}} a_{2}\right]=\left[\left(I-\Pi^{\mathrm{I}}\right) a_{2}\right]$. Equivalently

$$
-\left[\alpha_{2}\right] \Phi^{\mathrm{I}}+\alpha_{2}^{\mathrm{R}} \Phi^{\mathrm{R}}=\left[\left(I-\Pi^{\mathrm{I}}\right) a_{2}\right] .
$$

To compute the jump in the righthand side, use (3.17b) for the incident and transmitted $a_{1}$ (with the index ${ }^{\mathrm{I}}$ omitted as long as only incident and transmitted waves are involved),

$$
(I-\Pi) a_{2}=i Q\left(A_{1} \partial_{1}+\partial_{t}+A_{2} \partial_{2}+\sigma B\right) a_{1}
$$


Since $a_{1}$ is continuous on the interface, we obtain

$$
\left[(I-\Pi) a_{2}\right]=i Q\left(A_{1}\left[\partial_{1} a_{1}\right]+\sigma B a_{1}\right) \quad \text { on } x_{1}=0 .
$$

Split $a_{1}$ to compute the jump of derivatives,

$$
\left[\partial_{1} a_{1}\right]=\left[\partial_{1} \Pi a_{1}\right]+\left[\partial_{1}(I-\Pi) a_{1}\right]=\left[\partial_{1} \alpha_{1}\right] \Phi+\left[\partial_{1}(I-\Pi) a_{1}\right] .
$$

The jump of $\partial_{1} \alpha_{1}$ is obtained from the transport equation. Start with the jump of $\partial_{1}(I-$ П) $a_{1}$. From $B_{1} \Phi^{\mathrm{I}}=0$, and from (3.40), for both the incident and transmitted wave, we have

$$
(I-\Pi) a_{1}=i Q\left(\left(A_{2} \partial_{2}-\frac{1}{v_{1}} A_{1} \mathcal{T}\right) \alpha_{0} \Phi+\alpha_{0} A_{1} \Phi^{\prime}\right) .
$$

Differentiate (3.50) in $x_{1}$ and take the jump on the interface. Since the coefficients are smooth, only the jump of derivatives of $\alpha_{0}$ in $x_{1}$ intervene,

$$
\left[\partial_{1}(I-\Pi) a_{1}\right]=i Q\left(\left(A_{2} \partial_{2}-\frac{1}{v_{1}^{\mathrm{I}}} A_{1} \mathcal{T}\right)\left[\partial_{1} \alpha_{0}\right] \Phi+\left[\partial_{1} \alpha_{0}\right] A_{1} \Phi^{\prime}\right) .
$$

By (3.35), $\left[\partial_{1} \alpha_{0}\right]=-\frac{\sigma \gamma}{v_{1}} \alpha_{0}$, and since neither $\sigma$ nor $\varphi$ depend on the transverse variables, we obtain

$$
\left[\partial_{1}(I-\Pi) a_{1}\right]=-\frac{\sigma \gamma}{v_{1}}(I-\Pi) a_{1} \quad \text { on } x_{1}=0 .
$$

Next compute the transport equation for the incident and transmitted $a_{1}$. Begin with

$$
\Pi\left(\partial_{t}+A_{1} \partial_{1}+A_{2} \partial_{2}+\sigma B\right)\left(\alpha_{1} \Phi+(I-\Pi) a_{1}\right)=0 .
$$

The polarized part leads to the transport equation computed before, and we put the rest on the right hand side,

$$
\left(v_{1} \partial_{1} \alpha_{1}+\mathcal{T} \alpha_{1}+\sigma \gamma \alpha_{1}\right) \Phi=-\Pi\left(\partial_{t}+A_{2} \partial_{2}+A_{1} \partial_{1}+\sigma B\right)\left((I-\Pi) a_{1}\right) .
$$

Next take the jump on the interface. Since $(I-\Pi) a_{1}$ is continuous, we find

$$
\left(v_{1}\left[\partial_{1} \alpha_{1}\right]+\sigma \gamma \alpha_{1}\right) \Phi=-\Pi\left(A_{1}\left[\partial_{1}(I-\Pi) a_{1}\right]+\sigma B(I-\Pi) a_{1} \quad \text { on } x_{1}=0 .\right.
$$

Replace the first term after the equal sign from (3.51) to find

$$
\left(v_{1}\left[\partial_{1} \alpha_{1}\right]+\sigma \gamma \alpha_{1}\right) \Phi=-\sigma \Pi\left(-\frac{\gamma}{v_{1}} A_{1}+B\right)(I-\Pi) a_{1}=-\sigma \Pi B_{1}(I-\Pi) a_{1} .
$$

Since $B_{1}=L_{1}\left(x, d S^{\mathrm{I}}\right)=2(I-\Pi), \Pi B_{1}(I-\Pi)=0$, and therefore

$$
\left[\partial_{1} \Pi \alpha_{1}\right]=-\frac{\sigma \gamma}{v_{1}} \Pi a_{1} \text { on } x_{1}=0 .
$$

Adding (3.51) and (3.52) gives

$$
\left[\partial_{1} a_{1}\right]=-\frac{\sigma \gamma}{v_{1}} a_{1} \text { on } x_{1}=0 .
$$

Inserting in (3.49) gives, with a little algebra

$$
\left[(I-\Pi) a_{2}\right]=i \sigma Q B_{1} a_{1}=i \frac{\sigma}{2} L_{1} a_{1}=i \frac{\sigma}{2} L_{1}(I-\Pi) a_{1} .
$$


Insert now $(I-\Pi) a_{1}$ from (3.50):

$$
\left[(I-\Pi) a_{2}\right]=-\frac{\sigma}{2} L_{1} Q\left(\left(A_{2} \partial_{2}-\frac{1}{v_{1}} A_{1} \mathcal{T}\right) \alpha_{0} \Phi+\alpha_{0} A_{1} \Phi^{\prime}\right):=-\frac{\sigma}{4} L_{1} V .
$$

The righthand side is rearranged as

$$
V=\left(A_{2}-\frac{v_{2}}{v_{1}} A_{1}\right) \partial_{2} \alpha_{0} \Phi+\alpha_{0} A_{1}\left(\Phi^{\prime}-\frac{d}{v_{1}} \Phi\right) .
$$

It is not difficult to see that

$$
A_{1}\left(\Phi^{\prime}-\frac{d}{v_{1}} \Phi\right)=\frac{k c^{\prime}}{2 c \varphi^{\prime 2}} \Psi, \quad\left(A_{2}-\frac{v_{2}}{v_{1}} A_{1}\right) \Phi=-\frac{1}{\varphi^{\prime}} \Psi
$$

Therefore

$$
V=\left(\frac{k c^{\prime}}{2 c \varphi^{\prime 2}} \alpha_{0}-\frac{1}{\varphi^{\prime}} \partial_{2} \alpha_{0}\right) \Psi
$$

Since $L_{1} \Psi=L_{1}^{2} e_{1}=2 L_{1} e_{1}=2 \Psi$, we get

$$
\left[(I-\Pi) a_{2}\right]=-\frac{\sigma}{2}\left(\frac{k c^{\prime}}{2 c \varphi^{\prime 2}} \alpha_{0}-\frac{1}{\varphi^{\prime}} \partial_{2} \alpha_{0}\right) \Psi .
$$

Insert into (3.48) to obtain with the superscripts inserted

$$
-\left[\alpha_{2}\right] \Phi^{\mathrm{I}}+\alpha_{2}^{\mathrm{R}} \Phi^{\mathrm{R}}=-\frac{\sigma}{2}\left(\frac{k c^{\prime}}{2 c \varphi^{\prime 2}} \alpha_{0}^{\mathrm{I}}-\frac{1}{\varphi^{\mathrm{I}^{\prime}}} \partial_{2} \alpha_{0}^{\mathrm{I}}\right) \Psi^{\mathrm{I}} .
$$

The usual $\Psi^{\mathrm{I}}$. argument yields

$$
\alpha_{2}^{\mathrm{R}}=\frac{\sigma}{2 c \varphi^{\prime 2}}\left(\frac{k c^{\prime}}{2 c \varphi_{+}^{\prime}} \alpha_{0}^{\mathrm{I}}+\partial_{2} \alpha_{0}^{\mathrm{I}}\right)
$$

This completes the proof of Theorem 3.2.

\section{Reflection analysis for Bérenger's doubled system}

Bérenger's model is defined by doubling the equations as in (1.2), with $\sigma_{2} \equiv 0$ and $\sigma_{1}\left(x_{1}\right) \equiv$ $\sigma$, positive constant.

$$
\begin{aligned}
\widetilde{L}_{1} & =\partial_{t}+\widetilde{A}_{1} \partial_{1}+\widetilde{A}_{2} \partial_{2} \quad \text { and } \widetilde{L}=\widetilde{L}_{1}+\sigma \widetilde{B} \\
\text { with } & \widetilde{A}_{1}=\left(\begin{array}{cc}
A_{1} & A_{1} \\
0 & 0
\end{array}\right), \quad \widetilde{A}_{2}=\left(\begin{array}{cc}
0 & 0 \\
A_{2} & A_{2}
\end{array}\right), \quad \widetilde{B}=\left(\begin{array}{ll}
I & 0 \\
0 & 0
\end{array}\right) .
\end{aligned}
$$

When $\sigma=0$, the system is equivalent to the wave equation (3.1) (see [16] ).

In this section we perform a WKB analysis for the Cauchy problem with $\sigma>0$, with tools as in Section 3.2. Then we study the reflection and transmission in a medium with $\sigma=0$ for $x_{1}<0$, and $\sigma>0$ for $x_{1}>0$. 


\subsection{Asymptotic solutions for Bérenger's doubled system}

The ansatz for the free space solution of $\widetilde{L} u=0$ is

$$
\tilde{u}^{\varepsilon} \sim e^{i \tilde{S} / \varepsilon} \sum_{j=0}^{+\infty} \varepsilon^{j} \tilde{a}_{j}(t, x), \quad \text { with the phase } \quad \tilde{S}(t, x)=t+\tilde{\varphi}\left(x_{1}\right)+k x_{2} .
$$

Compute

$$
\widetilde{L}_{1}(x, d \tilde{S})=I+\tilde{\varphi}^{\prime} \widetilde{A}_{1}+k \widetilde{A}_{2}=\left(\begin{array}{cc}
I+\tilde{\varphi}^{\prime} A_{1} & \tilde{\varphi}^{\prime} A_{1} \\
k A_{2} & I+k A_{2}
\end{array}\right) .
$$

It follows that

$$
\widetilde{L}_{1}(x, d \tilde{S})\left(\begin{array}{l}
\Phi_{1} \\
\Phi_{2}
\end{array}\right)=L_{1}(x, d \tilde{S})\left(\Phi_{1}+\Phi_{2}\right)
$$

Therefore the phases are the same as for the original system. The ansatz is

$$
\tilde{u}^{\varepsilon} \sim e^{i S / \varepsilon} \sum_{j=0}^{+\infty} \varepsilon^{j} \tilde{a}_{j}(t, x), \quad \text { with the phase } S(t, x)=t+\varphi\left(x_{1}\right)+k x_{2} .
$$

The following algebraic results were established in [16]. By (4.2), the kernel of $\widetilde{L}_{1}$ is of dimension 1, spanned by

$$
\widetilde{\Phi}=\left(\varphi^{\prime} A_{1} \Phi, k A_{2} \Phi\right) .
$$

The matrix $\underset{\widetilde{Q}}{\widetilde{\Pi}}$ of the projector on $\operatorname{Ker} \widetilde{L}_{1}(x, d S)$, and $\widetilde{Q}$ the partial inverse of $\widetilde{L}_{1}(x, d S)$ defined by $\widetilde{Q} \widetilde{\Pi}=0$ and $\widetilde{Q} \widetilde{L}_{1}(x, d S)=I-\widetilde{\Pi}$, are obtained through the projector $\Pi$ on $\operatorname{Ker} L_{1}(x, d S)$ and the partial inverse $Q$ of $L_{1}(x, d S)$ in (3.24):

$$
\widetilde{\Pi}=-\left(\begin{array}{cc}
\varphi^{\prime} A_{1} \Pi & \varphi^{\prime} A_{1} \Pi \\
k A_{2} \Pi & k A_{2} \Pi
\end{array}\right), \quad \widetilde{Q}=\left(\begin{array}{cc}
I-\varphi^{\prime} A_{1} Q & -\varphi^{\prime} A_{1} Q \\
-k A_{2} Q & I-k A_{2} Q
\end{array}\right) .
$$

Theorem 4.1. With the same assumptions as in Theorem 3.1, the same results hold for the Bérenger's operator, and the principal term $\tilde{a}_{0}(t, x)=\tilde{\alpha}_{0} \widetilde{\Phi}$ is a solution of the transport equation

$$
\partial_{t} \tilde{\alpha}_{0}+\boldsymbol{v} \cdot \nabla \tilde{\alpha}_{0}+(d+\tilde{\gamma} \sigma) \tilde{\alpha}_{0}=0
$$

with $\boldsymbol{v}$ the group velocity defined in (3.6), d the zero order coefficients defined in (3.8), and $\tilde{\gamma}=c^{2} \varphi^{\prime 2}$.

Remark 4.1. The absorption coefficient $\tilde{\gamma}$ is the same as for the tuned layer.

Proof. The recursion relations are the same as in (3.17), adding a tilde when necessary. The coefficient $\tilde{a}_{0}$ is polarized, and $\tilde{a}_{0}=\widetilde{\Pi} \tilde{a}_{0}=\tilde{\alpha}_{0} \widetilde{\Phi}$. Next calculate the transport equation for $\tilde{\alpha}_{0}$. Write

$$
\widetilde{\Pi}\left(\partial_{t}+\widetilde{A}_{1} \partial_{1}+\widetilde{A}_{2} \partial_{2}+\sigma \widetilde{B}\right)\left(\tilde{\alpha}_{0} \widetilde{\Phi}\right)=0
$$

as

$$
\partial_{t} \tilde{\alpha}_{0} \widetilde{\Phi}+\partial_{1} \tilde{\alpha}_{0} \widetilde{\Pi} \widetilde{A}_{1} \widetilde{\Phi}+\partial_{2} \tilde{\alpha}_{0} \widetilde{\Pi} \widetilde{A}_{2} \widetilde{\Phi}+\left(\widetilde{\Pi} \widetilde{A}_{1} \widetilde{\Phi}^{\prime}+\sigma \widetilde{\Pi} \widetilde{B} \widetilde{\Phi}\right) \tilde{\alpha}_{0}=0
$$

Compute

$$
\widetilde{A}_{1} \widetilde{\Phi}=\left(\begin{array}{c}
-A_{1} \Phi \\
0
\end{array}\right), \quad \widetilde{\Pi} \widetilde{A}_{1} \widetilde{\Phi}=\left(\begin{array}{c}
\varphi^{\prime} A_{1} \Pi A_{1} \Phi \\
k A_{2} \Pi A_{1} \Phi
\end{array}\right)=\left(\begin{array}{c}
v_{1} A_{1} \Phi \\
v_{1} A_{2} \Phi
\end{array}\right)=v_{1} \widetilde{\Phi}
$$


by (3.18). $\widetilde{\Pi} \widetilde{A}_{2} \widetilde{\Phi}$ is computed in the same way, and we find

$$
\widetilde{\Pi} \widetilde{A}_{1} \widetilde{\Phi}=v_{1} \widetilde{\Phi}, \quad \widetilde{\Pi} \widetilde{A}_{2} \widetilde{\Phi}=v_{2} \widetilde{\Phi} .
$$

Next compute the term containing $\widetilde{\Phi}^{\prime}$ :

$$
\widetilde{\Pi} \widetilde{A}_{1} \widetilde{\Phi}^{\prime}=\widetilde{\Pi}\left(\begin{array}{c}
A_{1}\left(\left(\varphi^{\prime} A_{1}+k A_{2}\right) \Phi\right)^{\prime} \\
0
\end{array}\right) \stackrel{(4.4)}{=} \widetilde{\Pi}\left(\begin{array}{c}
A_{1}\left(-\Phi^{\prime}\right) \\
0
\end{array}\right) \stackrel{(4.5)}{=}\left(\begin{array}{c}
\varphi^{\prime} A_{1} \Pi A_{1} \Phi^{\prime} \\
k A_{2} \Pi A_{1} \Phi^{\prime}
\end{array}\right) .
$$

By (3.8), $\Pi A_{1} \Phi^{\prime}=d \Phi$, and so

$$
\widetilde{\Pi} \widetilde{A_{1}} \widetilde{\Phi}^{\prime}=d \widetilde{\Phi} .
$$

The last term $\widetilde{\Pi} \widetilde{B} \widetilde{\Phi}$ is computed using that $\Pi A_{1} \Phi=v_{1} \Phi$, and $v_{1}=-c^{2} \varphi^{\prime}$,

$$
\widetilde{\Pi} \widetilde{B} \widetilde{\Phi}=\widetilde{\Pi}\left(\begin{array}{c}
\varphi^{\prime} A_{1} \Phi \\
0
\end{array}\right)=-\varphi^{\prime}\left(\begin{array}{c}
\varphi^{\prime} A_{1} \Pi A_{1} \Phi \\
k A_{2} \Pi A_{1} \Phi
\end{array}\right)=-\varphi^{\prime} v_{1}\left(\begin{array}{c}
\varphi^{\prime} A_{1} \Phi \\
k A_{2} \Phi
\end{array}\right)=-\varphi^{\prime} v_{1} \widetilde{\Phi}=c^{2} \varphi^{\prime 2} \widetilde{\Phi} .
$$

Summarizing, we get the assertion in the theorem.

\subsection{Reflection coefficients for Bérenger's layer}

The input is an incident wave in $\left\{x_{1} \leq 0\right\}$ with phase $S^{\mathrm{I}}(t, x):=t-\varphi_{+}\left(x_{1}\right)+k x_{2}$,

$$
\tilde{u}^{\varepsilon}:=e^{i S^{\mathrm{I}}(t, x) / \varepsilon} \tilde{a}^{\mathrm{I}}(t, x, \varepsilon), \quad \tilde{a}^{\mathrm{I}}(t, x, \varepsilon) \sim \sum_{j=0}^{\infty} \varepsilon^{j} \tilde{a}_{j}^{\mathrm{I}}(t, x), \quad \widetilde{L}_{1}\left(\partial_{t}, \partial_{x}\right) \tilde{u}^{\varepsilon}=O\left(\varepsilon^{\infty}\right) .
$$

Suppose that the amplitudes $\tilde{a}_{j}^{\mathrm{I}}$ are supported in a tube $T$ of rays with compact temporal cross sections $T \cap\{t=0\} \subset \subset\left\{x_{1}<0\right\}$.

By Theorem 4.1, we construct a transmitted wave with the same phase, and a reflected wave with phase $S^{\mathrm{R}}(t, x):=t+\varphi_{+}\left(x_{1}\right)+k x_{2}$. The reflected wave $\tilde{v}^{\varepsilon}$ is supported in $x_{1} \leq 0$ :

$$
\tilde{v}^{\varepsilon}=e^{i S^{\mathrm{R}}(t, x) / \varepsilon} \tilde{a}^{\mathrm{R}}(t, x, \varepsilon), \quad \tilde{a}^{\mathrm{R}}(t, x, \varepsilon) \sim \sum_{j=0}^{\infty} \varepsilon^{j} \tilde{a}_{j}^{\mathrm{R}}(t, x), \quad \widetilde{L}_{1}\left(\partial_{t}, \partial_{x}\right) v^{\varepsilon}=O\left(\varepsilon^{\infty}\right) .
$$

The transmitted wave is supported in $x_{1} \geq 0$,

$$
\tilde{w}^{\varepsilon}:=e^{i S^{\mathrm{I}}(t, x) / \varepsilon} \tilde{a}^{\mathrm{T}}(t, x, \varepsilon), \quad \tilde{a}^{\mathrm{T}}(t, x, \varepsilon) \sim \sum_{j=0}^{\infty} \varepsilon^{j} \tilde{a}_{j}^{\mathrm{T}}(t, x), \quad L\left(\partial_{t}, \partial_{x}\right) \tilde{w}^{\varepsilon}=O\left(\varepsilon^{\infty}\right) .
$$

\section{Theorem 4.2.}

(i) Given the incoming amplitudes $\tilde{a}_{j}^{I}$ there are uniquely determined amplitudes $\tilde{a}_{j}^{T}$ and $\tilde{a}_{j}^{R}$ so that $\tilde{u}^{\varepsilon}, \tilde{v}^{\varepsilon}$, and $\tilde{w}^{\varepsilon}$ are infinitely accurate solutions of the differential equations and the transmission condition is also satisfied to infinite order,

$$
\forall\left(t, x_{2}\right) \in \mathbb{R} \times \mathbb{R}, \quad \widetilde{A}_{1}\left(\tilde{u}^{\varepsilon}+\tilde{v}^{\varepsilon}\right)\left(t, 0_{-}, x_{2}\right)=\widetilde{A}_{1} \tilde{w}^{\varepsilon}\left(t, 0_{+}, x_{2}\right)+O\left(\varepsilon^{\infty}\right) .
$$

(ii) If $\sigma\left(x_{1}\right)=\sigma \mathbf{1}_{x_{1}>0}$, the coefficients $\tilde{a}_{0}^{R}$ and $\tilde{a}_{1}^{R}$ vanishes identically. The reflection coefficient of the layer is equal to

$$
R(k)=-\frac{\sigma k c^{\prime}(0)}{4 c^{2}(0) \varphi_{+}^{\prime 3}(0)} .
$$

That is, noting $\tilde{a}_{0}^{I}(t, x)=\tilde{\alpha}_{0}^{I}(t, x) \widetilde{\Phi}^{I} \in \operatorname{Ker} \widetilde{L}_{1}\left(0, d S^{I}\right)$, then

$$
\tilde{a}_{2}^{R}\left(t, 0_{-}, x_{2}\right)=R(k) \tilde{\alpha}_{0}^{I}\left(t, 0_{-}, x_{2}\right) \widetilde{\Phi}^{R} .
$$


Remark 4.2. Bérenger's layer with $\sigma\left(x_{1}\right)$ satisfying $\sigma(0)=\cdots=\sigma^{(p-1)}(0)=0, \sigma^{(p)}(0) \neq$ 0 is nonreflecting at order $p+1$. There exists $R_{p}(k) \neq 0$ such that

$$
a_{p+2}^{R}\left(t, 0_{-}, x_{2}\right)=\sigma^{(p)}(0) R_{p}(k) \tilde{\alpha}_{0}^{I}\left(t, 0_{-}, x_{2}\right) \widetilde{\Phi}^{R} .
$$

The proof of this case follows the proof of Theorem 6.1.(iv) in [16].

Proof of Theorem. Follows that of the preceding section. We will show that the zero and first order terms in the expansion of the reflected wave vanish in $x_{1}<0$, that $\tilde{a}_{2}^{\mathrm{R}}(t, x)=\tilde{\alpha}_{2}^{\mathrm{R}}(t, x) \widetilde{\Phi}^{\mathrm{R}}$, and express $\tilde{\alpha}_{2}^{\mathrm{R}}$ in terms of the incident wave. Define, for every $j$, $\tilde{\alpha}_{j}$ by

$$
\widetilde{\Pi}^{\mathrm{I}} \tilde{a}_{j}^{\mathrm{I}, \mathrm{T}}=\tilde{\alpha}_{j}^{\mathrm{I}, \mathrm{T}} \widetilde{\Phi}^{\mathrm{I}}, \quad \widetilde{\Pi}^{\mathrm{R}} \tilde{a}_{j}^{\mathrm{R}}=\tilde{\alpha}_{j}^{\mathrm{R}} \widetilde{\Phi}^{\mathrm{R}} .
$$

Compute the transmission at the interface. Since the coefficients of order 0 are all polarized, one has $\tilde{\alpha}_{0}^{\mathrm{I}} \widetilde{\Phi}^{\mathrm{I}}+\tilde{\alpha}_{0}^{\mathrm{R}} \widetilde{\Phi}^{\mathrm{R}}=\tilde{\alpha}_{0}^{\mathrm{T}} \widetilde{\Phi}^{\mathrm{I}}$ on $x_{1}=0$. Since the vectors $\widetilde{\Phi}^{\mathrm{I}}$ and $\widetilde{\Phi}^{\mathrm{R}}$ are transverse, this yields

$$
\tilde{\alpha}_{0}^{\mathrm{R}}=0 \quad \text { and } \tilde{\alpha}_{0}^{\mathrm{T}}=\tilde{\alpha}_{0}^{\mathrm{I}} \text { on } x_{1}=0 .
$$

Using the group velocity $\boldsymbol{v}^{\mathrm{I}}\left(x_{1}\right)=c^{2}\left(\varphi_{+}^{\prime},-k\right)$, the transport equation for the incident, reflected and transmitted waves, with the transverse part (3.38) are

$$
\begin{array}{lll}
v_{1}^{\mathrm{I}} \partial_{1} \tilde{\alpha}_{0}^{\mathrm{I}}+\mathcal{T}^{\mathrm{I}} \tilde{\alpha}_{0}^{\mathrm{I}} & =0 & \\
v_{1}^{\mathrm{I}} \partial_{1} \tilde{\alpha}_{0}^{\mathrm{T}}+\mathcal{T}^{\mathrm{I}} \tilde{\alpha}_{0}^{\mathrm{T}}+\sigma \tilde{\gamma} \tilde{\alpha}_{0}^{\mathrm{T}} & =0, & \\
-v_{1}^{\mathrm{I}} \partial_{1} \tilde{\alpha}_{0}^{\mathrm{R}}+\mathcal{T}^{\mathrm{R}} \tilde{\alpha}_{0}^{\mathrm{R}}=0, & x_{1}<0 .
\end{array}
$$

Since the reflected wave vanishes at time 0 , the transport equation (4.12c) propagating to the left implies that $\tilde{\alpha}_{0}^{\mathrm{R}}$ is identically equal to zero in $\mathbb{R}_{-}^{2} \times \mathbb{R}$. Since $\tilde{\alpha}_{0}^{\mathrm{T}}=\tilde{\alpha}_{0}^{\mathrm{I}}$ on $x_{1}=0$, $\mathcal{T} \tilde{\alpha}_{0}^{\mathrm{T}}=\mathcal{T} \tilde{\alpha}_{0}^{\mathrm{I}}$. Subtracting (4.12a) from (4.12b), it follows that

$$
v_{1}^{\mathrm{I}}\left[\partial_{1} \tilde{\alpha}_{0}\right]+\sigma \tilde{\gamma} \tilde{\alpha}_{0}^{\mathrm{I}}=0 \quad \text { on } x_{1}=0 .
$$

Next compute the terms of order 1 with tildized (3.19) at order 1 ,

$$
(I-\widetilde{\Pi}) \tilde{a}_{1}^{\mathrm{I}, \mathrm{R}, \mathrm{T}}=i \widetilde{Q}\left(\widetilde{A}_{1} \partial_{1}+\widetilde{L}_{T}+\sigma \widetilde{B}\right) \tilde{\alpha}_{0}^{\mathrm{I}, \mathrm{R}, \mathrm{T}} \widetilde{\Phi}^{\mathrm{I}, \mathrm{R}, \mathrm{I}}, \quad \widetilde{L}_{T}:=\partial_{t}+\widetilde{A}_{2} \partial_{2} .
$$

Since $\tilde{\alpha}_{0}^{\mathrm{R}} \equiv 0$ in $\mathbb{R}_{-}^{2} \times \mathbb{R},(I-\widetilde{\Pi}) \tilde{a}_{1}^{\mathrm{R}} \equiv 0$ in $\mathbb{R}_{-}^{2} \times \mathbb{R}$. For the incident and transmitted waves (indices ${ }^{\mathrm{I}, \mathrm{T}}$ omitted), replace $\partial_{1} \tilde{\alpha}_{0}$ from (4.12), with $\sigma=0$ for the incident wave,

$$
\begin{aligned}
(I-\widetilde{\Pi}) \tilde{a}_{1} & =i \widetilde{Q}\left(\left(-\frac{1}{v_{1}^{\mathrm{I}}} \widetilde{A}_{1}\left(\mathcal{T} \tilde{\alpha}_{0}+\sigma \tilde{\gamma} \tilde{\alpha}_{0}\right)+\left(\widetilde{L}_{T}+\sigma \widetilde{B}\right) \tilde{\alpha}_{0}\right) \widetilde{\Phi}+\tilde{\alpha}_{0} \widetilde{A}_{1} \widetilde{\Phi}^{\prime}\right) \\
& =i \widetilde{Q}\left(\left(\left(\widetilde{L}_{T}-\frac{1}{v_{1}^{\mathrm{I}}} \widetilde{A}_{1} \mathcal{T}\right) \tilde{\alpha}_{0}+\left(\sigma \widetilde{B}-\frac{\sigma \tilde{\gamma}}{v_{1}^{\mathrm{I}}} \widetilde{A}_{1}\right) \tilde{\alpha}_{0}\right) \widetilde{\Phi}+\tilde{\alpha}_{0} \widetilde{A}_{1} \widetilde{\Phi}^{\prime}\right) \\
& =i \widetilde{Q}\left(\left(\left(\widetilde{L}_{T}-\frac{1}{v_{1}^{\mathrm{I}}} \widetilde{A}_{1} \mathcal{T}\right) \tilde{\alpha}_{0}+\sigma \widetilde{B}_{1} \tilde{\alpha}_{0}\right) \widetilde{\Phi}+\tilde{\alpha}_{0} \widetilde{A}_{1} \widetilde{\Phi}^{\prime}\right),
\end{aligned}
$$

with

$$
\widetilde{B}_{1}=\widetilde{B}-\frac{\tilde{\gamma}}{v_{1}^{\mathrm{I}}} \widetilde{A}_{1}=\widetilde{B}+\varphi^{\mathrm{I}} \widetilde{A}_{1}
$$

We first claim that the term in $\sigma$ vanishes, since (omitting the ${ }^{\mathrm{I}}$ 's)

$$
\widetilde{B}_{1} \widetilde{\Phi}=\left(\begin{array}{c}
\varphi^{\prime} A_{1} \Phi+\varphi^{\prime} A_{1}\left(\varphi^{\prime} A_{1}+k A_{2}\right) \Phi \\
0
\end{array}\right)=\left(\begin{array}{c}
\varphi^{\prime} A_{1} \Phi-\varphi^{\prime} A_{1} \Phi \\
0
\end{array}\right)=0
$$


and therefore for both the incident and transmitted waves,

$$
(I-\widetilde{\Pi}) \tilde{a}_{1}=i \widetilde{Q}\left(\left(\widetilde{L}_{T}-\frac{1}{v_{1}^{\mathrm{I}}} \widetilde{A}_{1} \mathcal{T}\right) \tilde{\alpha}_{0} \widetilde{\Phi}+\tilde{\alpha}_{0} \widetilde{A}_{1} \widetilde{\Phi}^{\prime}\right)
$$

Since the previous equation does not contain $\sigma$, it shows that on the interface $\left[(I-\widetilde{\Pi}) \tilde{a}_{1}\right]=$ 0 . Summarizing, for the reflected wave in $\mathbb{R}_{-}^{2},\left(I-\widetilde{\Pi}^{\mathrm{R}}\right) \tilde{a}_{1}^{\mathrm{R}} \equiv 0$, for the incoming wave in $\mathbb{R}_{-}^{2}$ and the transmitted wave in $\mathbb{R}_{+}^{2},\left[(I-\widetilde{\Pi}) \tilde{a}_{1}\right]=0$. The transmission condition for $a_{1}$ is

$$
\tilde{\alpha}_{1}^{\mathrm{R}} \widetilde{\Phi}^{\mathrm{R}}+\left[\tilde{\alpha}_{1}\right] \widetilde{\Phi}^{\mathrm{I}}=\left[\left(I-\widetilde{\Pi}^{\mathrm{I}}\right) \tilde{a}_{1}\right]=0 \quad \text { on } x_{1}=0,
$$

and shows that

$$
\tilde{\alpha}_{1}^{\mathrm{R}}=0 \quad \text { and } \tilde{\alpha}_{1}^{\mathrm{T}}=\tilde{\alpha}_{1}^{\mathrm{I}} \text { on } x_{1}=0 .
$$

Next compute the transport equation for $\tilde{\alpha}_{1}^{\mathrm{R}}$. Since $\left(I-\widetilde{\Pi}^{\mathrm{R}}\right) \tilde{\alpha}_{1}^{\mathrm{R}} \equiv 0$ in $\mathbb{R}_{-}^{d},(4.12 \mathrm{c})$ is valid for $\tilde{\alpha}_{1}^{\mathrm{R}}$ :

$$
\partial_{t} \tilde{\alpha}_{1}^{\mathrm{R}}+\boldsymbol{v}^{\mathrm{R}} \cdot \nabla \tilde{\alpha}_{1}^{\mathrm{R}}+d^{\mathrm{R}} \tilde{\alpha}_{1}^{\mathrm{R}}=0 .
$$

Again, $\tilde{\alpha}_{1}^{\mathrm{R}}$ being solution of the leftward transport equation with zero boundary and initial data, vanishes identically in $\mathbb{R}_{-}^{2}$. Summarizing the information obtained so far,

$$
\tilde{a}_{1}^{\mathrm{R}}=\widetilde{\Pi}^{\mathrm{R}} \tilde{a}_{1}^{\mathrm{R}} \equiv 0 \text { in } \mathbb{R}_{-}^{2}, \quad\left[\tilde{a}_{1}\right]=0 \text { on } x_{1}=0 .
$$

To calculate the next term in the expansion of the reflected wave, start with the transmission condition $\tilde{a}_{2}^{\mathrm{I}}+\tilde{a}_{2}^{\mathrm{R}}=\tilde{a}_{2}^{\mathrm{T}}$ on $x_{1}=0$. Split into polarized and not polarized parts,

$$
\widetilde{\Pi}^{\mathrm{I}} \tilde{a}_{2}^{\mathrm{I}}+\widetilde{\Pi}^{\mathrm{R}} \tilde{a}_{2}^{\mathrm{R}}+\left(I-\widetilde{\Pi}^{\mathrm{I}}\right) \tilde{a}_{2}^{\mathrm{I}}+\left(I-\widetilde{\Pi}^{\mathrm{R}}\right) \tilde{a}_{2}^{\mathrm{R}}=\widetilde{\Pi}^{\mathrm{I}} \tilde{a}_{2}^{\mathrm{T}}+\left(I-\widetilde{\Pi}^{\mathrm{I}}\right) \tilde{a}_{2}^{\mathrm{T}} \quad \text { on } x_{1}=0 .
$$

By (4.16) and tildized (3.17b), $\left(I-\widetilde{\Pi}^{\mathrm{R}}\right) \tilde{a}_{2}^{\mathrm{R}} \equiv 0$ in $\mathbb{R}_{-}^{2}$, and the previous equation can be written as

$$
\widetilde{\Pi}^{\mathrm{R}} \tilde{a}_{2}^{\mathrm{R}}-\left[\widetilde{\Pi}^{\mathrm{I}} \tilde{a}_{2}\right]=\left[\left(I-\widetilde{\Pi}^{\mathrm{I}}\right) \tilde{a}_{2}\right] \quad \text { on } x_{1}=0,
$$

or equivalently

$$
\left[\tilde{\alpha}_{2}\right] \widetilde{\Phi}^{\mathrm{I}}+\tilde{\alpha}_{2}^{\mathrm{R}} \widetilde{\Phi}^{\mathrm{R}}=\left[\left(I-\widetilde{\Pi}^{\mathrm{I}}\right) \tilde{a}_{2}\right] \quad \text { on } x_{1}=0 .
$$

The vectors $\widetilde{\Phi}^{\mathrm{I}}$ and $\widetilde{\Phi}^{\mathrm{R}}$ are transverse, hence giving a unique solution $\left(\left[\tilde{\alpha}_{2}\right], \tilde{\alpha}_{2}^{\mathrm{R}}\right)$. Take the scalar product with $\widetilde{\Psi}=\left(\Psi^{\mathrm{I}}, \Psi^{\mathrm{I}}\right)$. Since $\left(\Phi^{\mathrm{I}}, \Psi^{\mathrm{I}}\right)=0$, then $\left(\widetilde{\Phi}^{\mathrm{I}}, \widetilde{\Psi}^{\mathrm{I}}\right)=0$, and we find

$$
\tilde{\alpha}_{2}^{\mathrm{R}}=\frac{\left(\left[\left(I-\widetilde{\Pi}^{\mathrm{I}}\right) \tilde{a}_{2}\right], \widetilde{\Psi}\right)}{\left(\widetilde{\Phi}^{\mathrm{R}}, \widetilde{\Psi}\right)}=\frac{\left(\left[\left(I-\widetilde{\Pi}^{\mathrm{I}}\right) \tilde{a}_{2}\right], \widetilde{\Psi}\right)}{\left(\Phi^{\mathrm{R}}, \Psi^{\mathrm{I}}\right)} .
$$

To compute the jump in the righthand side, use (3.17b), omitting the indices ${ }^{\mathrm{I}}$,

$$
(I-\widetilde{\Pi}) \tilde{a}_{2}=i \widetilde{Q}\left(\widetilde{A}_{1} \partial_{1}+\widetilde{L}_{T}+\sigma \widetilde{B}\right) \tilde{a}_{1} .
$$

Since $\tilde{a}_{1}$ is continuous on the interface, we obtain

$$
\left[(I-\widetilde{\Pi}) \tilde{a}_{2}\right]=i \widetilde{Q}\left(\widetilde{A}_{1}\left[\partial_{1} \tilde{a}_{1}\right]+\sigma \widetilde{B} \tilde{a}_{1}\right) .
$$

Split again $\tilde{a}_{1}$ to compute the jump of derivatives:

$$
\left[\partial_{1} \tilde{a}_{1}\right]=\left[\partial_{1} \widetilde{\Pi} \tilde{a}_{1}\right]+\left[\partial_{1}(I-\widetilde{\Pi}) \tilde{a}_{1}\right]=\left[\partial_{1} \tilde{\alpha}_{1}\right] \widetilde{\Phi}+\left[\partial_{1}(I-\widetilde{\Pi}) \tilde{a}_{1}\right] .
$$


The jump of $\partial_{1} \tilde{\alpha}_{1}$ will be obtained from the transport equation, the jump of $\partial_{1}(I-\widetilde{\Pi}) \tilde{a}_{1}$ by differentiating (4.15) in $x_{1}$ and taking the jump on the interface. Start with the latter.

$$
\partial_{1}(I-\widetilde{\Pi}) \tilde{a}_{1}=\partial_{1}\left(i \widetilde{Q}\left(\left(\widetilde{L}_{T}-\frac{1}{v_{1}} \widetilde{A}_{1} \mathcal{T}\right) \tilde{\alpha}_{0} \widetilde{\Phi}+\frac{k c^{\prime}}{2 c \varphi^{\prime 2}} \tilde{\alpha}_{0}\left(\begin{array}{c}
\Psi \\
0
\end{array}\right)\right)\right) .
$$

Since the coefficients are smooth, only the jump of derivatives of $\tilde{\alpha}_{0}$ intervenes:

$$
\left[\partial_{1}(I-\widetilde{\Pi}) \tilde{a}_{1}\right]=i \widetilde{Q}\left(\left(\widetilde{L}_{T}-\frac{1}{v_{1}^{\mathrm{I}}} \widetilde{A}_{1} \mathcal{T}\right)\left[\partial_{1} \tilde{\alpha}_{0}\right] \widetilde{\Phi}+\frac{k c^{\prime}}{2 c \varphi^{\prime 2}}\left[\partial_{1} \tilde{\alpha}_{0}\right]\left(\begin{array}{c}
\Psi \\
0
\end{array}\right)\right)
$$

By (4.13), $\left[\partial_{1} \tilde{\alpha}_{0}\right]=-\frac{\sigma \tilde{\gamma}}{v_{1}^{\mathrm{I}}} \tilde{\alpha}_{0}^{\mathrm{I}}=\sigma \varphi_{+}^{\prime} \tilde{\alpha}_{0}^{\mathrm{I}}$, and since neither $\sigma$ nor $\varphi$ depend on the transverse variables, we obtain

$$
\left[\partial_{1}(I-\widetilde{\Pi}) \tilde{a}_{1}\right]=\sigma \varphi_{+}^{\prime}(I-\widetilde{\Pi}) \tilde{a}_{1} \quad \text { on } x_{1}=0 .
$$

Next compute the transport equation for the incident and transmitted $\tilde{\alpha}_{1}$. Begin with

$$
\widetilde{\Pi}\left(\partial_{t}+\widetilde{A}_{1} \partial_{1}+\widetilde{A}_{2} \partial_{2}+\sigma \widetilde{B}\right)\left(\tilde{\alpha}_{1} \widetilde{\Phi}+(I-\widetilde{\Pi}) \tilde{a}_{1}\right)=0 .
$$

The polarized part leads to the transport equation computed before, and we put the rest in the righthand side:

$$
\left(v_{1} \partial_{1} \tilde{\alpha}_{1}+\mathcal{T} \tilde{\alpha}_{1}+\sigma \tilde{\gamma} \tilde{\alpha}_{1}\right) \widetilde{\Phi}=-\widetilde{\Pi}\left(\widetilde{L}_{T}+\widetilde{A}_{1} \partial_{1}+\sigma \widetilde{B}\right)\left((I-\widetilde{\Pi}) \tilde{a}_{1}\right) .
$$

Next take the jump on the interface. Since $(I-\widetilde{\Pi}) \tilde{a}_{1}$ is continuous, we find

$$
\left(v_{1}\left[\partial_{1} \tilde{\alpha}_{1}\right]+\sigma \tilde{\gamma} \tilde{\alpha}_{1}\right) \widetilde{\Phi}=-\widetilde{\Pi}\left(\widetilde{A}_{1}\left[\partial_{1}(I-\widetilde{\Pi}) \tilde{a}_{1}\right]+\sigma \widetilde{B}(I-\widetilde{\Pi}) \tilde{a}_{1}\right) .
$$

Replace the first term after the equal sign from (4.19) with the help of (4.14), to find

$$
\left(v_{1}\left[\partial_{1} \tilde{\alpha}_{1}\right]+\sigma \tilde{\gamma} \tilde{\alpha}_{1}\right) \widetilde{\Phi}=-\sigma \widetilde{\Pi}\left(-\frac{\tilde{\gamma}}{v_{1}} \widetilde{A}_{1}+\widetilde{B}\right)(I-\widetilde{\Pi}) \tilde{a}_{1}=-\sigma \widetilde{\Pi} \widetilde{B}_{1}(I-\widetilde{\Pi}) \tilde{a}_{1} .
$$

We have already met the matrix $\widetilde{B}_{1}=\varphi^{\prime} \widetilde{A}_{1}+\widetilde{B}$ and shown that it vanishes on the kernel of $\widetilde{L}_{1}(x, d S)$. Therefore

$$
\left[\partial_{1} \tilde{\alpha}_{1}\right] \widetilde{\Phi}==-\sigma\left(\frac{1}{v_{1}} \widetilde{\Pi} \widetilde{B}_{1}+\varphi^{\prime} \widetilde{\Pi}\right) \tilde{a}_{1}
$$

Defering (4.20) and (4.19) in (4.18) gives, with a little algebra,

$$
\left[(I-\widetilde{\Pi}) \tilde{a}_{2}\right]=i \sigma \widetilde{Q}\left(I-\frac{1}{v_{1}} \widetilde{A}_{1} \widetilde{\Pi}\right) \widetilde{B}_{1} \tilde{a}_{1}=i \sigma \widetilde{Q}\left(I-\frac{1}{v_{1}} \widetilde{A}_{1} \widetilde{\Pi}\right) \widetilde{B}_{1}(I-\widetilde{\Pi}) \tilde{a}_{1} .
$$

$(I-\widetilde{\Pi}) \tilde{a}_{1}$ was computed in $(4.15)$ as

$$
(I-\widetilde{\Pi}) \tilde{a}_{1}=i \widetilde{Q}(\widetilde{V}+\widetilde{W}), \quad \widetilde{V}:=\left(\widetilde{L}_{T}-\frac{1}{v_{1}} \widetilde{A}_{1} \mathcal{T}\right) \tilde{\alpha}_{0} \widetilde{\Phi}, \quad \widetilde{W}:=\frac{k c^{\prime}}{2 c \varphi^{\prime 2}} \tilde{\alpha}_{0}\left(\begin{array}{l}
\Psi \\
0
\end{array}\right) .
$$

and therefore

$$
\left[(I-\widetilde{\Pi}) \tilde{a}_{2}\right]=-\sigma \widetilde{Q}\left(I-\frac{1}{v_{1}} \widetilde{A}_{1} \widetilde{\Pi}\right) \widetilde{B}_{1} \widetilde{Q}(\widetilde{V}+\widetilde{W}) .
$$

Plugging into (4.17), reintroducing the exponents ${ }^{\mathrm{I}}$, we find 


$$
\tilde{\alpha}_{2}^{\mathrm{R}}=\frac{\left(-\sigma \widetilde{Q}^{\mathrm{I}}\left(I-\frac{1}{v_{1}^{\mathrm{I}}} \widetilde{A}_{1} \widetilde{\Pi}^{\mathrm{I}}\right) \widetilde{B}_{1} \widetilde{Q}^{\mathrm{I}}(\widetilde{V}+\widetilde{W}), \widetilde{\Psi}\right)}{\left(\Phi^{\mathrm{R}}, \Psi^{\mathrm{I}}\right)} .
$$

We know from perfect matching that for constant coefficients $\left(c^{\prime} \equiv 0, \widetilde{W}=0\right)$, the PML is perfectly matched. In particular on the interface $\tilde{\alpha}_{2}^{\mathrm{R}}$ is zero. Therefore the contribution of $\widetilde{V}$ vanishes, and only the term containing $\widetilde{W}$ remains. To compute the coefficient, we need an intermediate technical computation.

Claim. For any vector $\widetilde{V}=\left(V_{1}, V_{2}\right)$,

$$
\left(I-\frac{1}{v_{1}} \widetilde{A}_{1} \widetilde{\Pi}\right)\left(\widetilde{B}-\frac{\gamma}{v_{1}} \widetilde{A}_{1}\right) \widetilde{Q} \widetilde{V}=\left(\begin{array}{c}
\left(I-\frac{1}{v_{1}} A_{1} \Pi\right) V_{1} \\
0
\end{array}\right)
$$

Proof of Claim. First the equality

$$
\left(\widetilde{B}-\frac{\gamma}{v_{1}} \widetilde{A}_{1}\right) \widetilde{Q} \widetilde{V}=\left(\begin{array}{c}
V_{1}+\varphi^{\prime} A_{1} \Pi\left(V_{1}+V_{2}\right) \\
0
\end{array}\right)
$$

is obtained by explicit calculations, using that $\varphi^{\prime} A_{1}+k A_{2}=L_{1}-I, Q=\frac{1}{4} L_{1}$ and $\Pi=I-2 L_{1}$. Then some manipulations show that

$$
\widetilde{A}_{1} \widetilde{\Pi}\left(\begin{array}{c}
V_{1}+\varphi^{\prime} A_{1} \Pi\left(V_{1}+V_{2}\right) \\
0
\end{array}\right)=\left(\begin{array}{c}
A_{1} \Pi\left(V_{1}+\varphi^{\prime} A_{1} \Pi\left(V_{1}+V_{2}\right)\right) \\
0
\end{array}\right)=\left(\begin{array}{c}
A_{1} \Pi V_{1}+\varphi^{\prime} v_{1} A_{1} \Pi\left(V_{1}+V_{2}\right) \\
0
\end{array}\right)
$$

Subtracting these two expressions proves the claim.

The claim implies that

$$
\left(I-\frac{1}{v_{1}^{\mathrm{I}}} \widetilde{A}_{1} \widetilde{\Pi}^{\mathrm{I}}\right) \widetilde{B}_{1} \widetilde{Q}^{\mathrm{I}}\left(\begin{array}{c}
\Psi^{\mathrm{I}} \\
0
\end{array}\right)=\left(\begin{array}{c}
\left(I-\frac{1}{v_{1}^{\mathrm{I}}} A_{1} \Pi\right) \Psi^{\mathrm{I}} \\
0
\end{array}\right)=\left(\begin{array}{c}
\Psi^{\mathrm{I}} \\
0
\end{array}\right) .
$$

Therefore

$$
\tilde{\alpha}_{2}^{\mathrm{R}}=-\sigma \frac{k c^{\prime}}{2 c \varphi^{\prime 2}} \frac{\left(\widetilde{Q}^{\mathrm{I}}\left(\begin{array}{c}
\Psi^{\mathrm{I}} \\
0
\end{array}\right), \widetilde{\Psi}\right)}{\left(\Phi^{\mathrm{R}}, \Psi^{\mathrm{I}}\right)} \tilde{\alpha}_{0}^{\mathrm{I}} .
$$

Compute the right hand side

$$
\begin{aligned}
\left(\widetilde{Q}^{\mathrm{I}}\left(\begin{array}{c}
\Psi^{\mathrm{I}} \\
0
\end{array}\right), \widetilde{\Psi}\right) & =\left(\begin{array}{c}
\left(I-\varphi^{\mathrm{I}} A_{1} Q^{\mathrm{I}}\right) \Psi^{\mathrm{I}} \\
-k A_{2} Q^{\mathrm{I}} \Psi^{\mathrm{I}}
\end{array}\right) \cdot\left(\begin{array}{c}
\Psi^{\mathrm{I}} \\
\Psi^{\mathrm{I}}
\end{array}\right) \\
& =\left(\left(I-\left(\varphi^{\mathrm{I}} A_{1}+k A_{2}\right) Q^{\mathrm{I}}\right) \Psi^{\mathrm{I}}, \Psi^{\mathrm{I}}\right) \\
& =\left(\left(I-\left(L^{\mathrm{I}}-I\right) Q^{\mathrm{I}}\right) \Psi^{\mathrm{I}}, \Psi^{\mathrm{I}}\right) \\
& =\left(\left(I-Q^{\mathrm{I}}\right) \Psi^{\mathrm{I}}, \Psi^{\mathrm{I}}\right)=\frac{1}{2}\left\|\Psi^{\mathrm{I}}\right\|^{2}
\end{aligned}
$$

since $Q^{\mathrm{I}} \Psi^{\mathrm{I}}=\frac{1}{2} \Psi^{\mathrm{I}}$. Therefore

$$
\tilde{\alpha}_{2}^{\mathrm{R}}=-\sigma \frac{k c^{\prime}}{4 c \varphi^{\prime 2}} \frac{\left\|\Psi^{\mathrm{I}}\right\|^{2}}{\left(\Psi^{\mathrm{I}}, \Phi^{\mathrm{R}}\right)} \tilde{\alpha}_{0}^{\mathrm{I}}=-\sigma \frac{k c^{\prime}}{4 c^{2} \varphi^{\prime 3}} \tilde{\alpha}_{0}^{\mathrm{I}}
$$




\section{$5 \quad$ Numerical experiments}

Numerical simulations in this section exhibit the reflections proved to exist in the asymptotic expansions. Their amplitudes as a function of frequency correspond to the leading order predictions of the theory. Since the theorems are rigorous and the accuracy of the numerics is checked, what this shows is that the asymptotic regime is attained for the frequencies tested. Figure 2 shows the interesting fact that with comparable wave numbers much smaller meshes are needed to resolve Bérenger's layer in the asymptotic regime.

The computational domain (see Figure 5 ) is the rectangle $\mathcal{D}:=]-1,1.6[\times]-2,2[$ (black box). The smaller rectangle of interest is $\mathcal{R}:=]-1, r[\times]-1,1[$ with $r=0.6$ (blue box). The magenta box is the support of the data. The absorbing layer is $] r, 1.6[\times]-2,2[$ in green. The domain $\mathcal{D}$ is wide in the $x_{1}$ and $x_{2}$ directions so that waves reflected from the external boundaries during the time of computation $T=2$ do not interfere with the reflections from the interface.

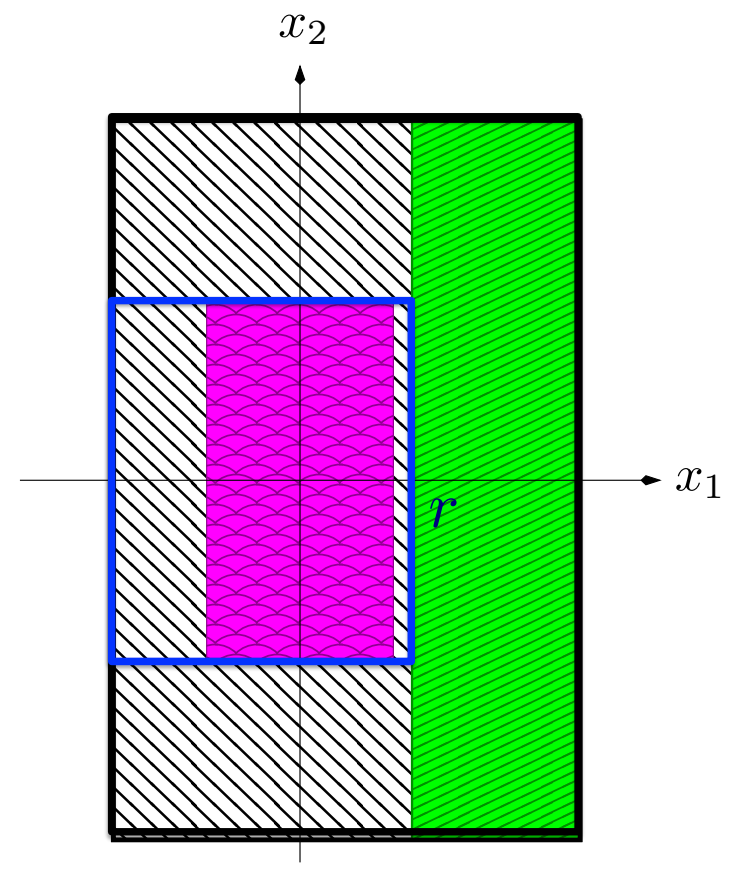

Figure 1: Data in the $x$ plane

A very precise numerical solution in $\mathcal{R}$ is computed using a large computational domain and small discretization size with an excessively large computation cost. The error in $\mathcal{R}$ is the difference between the solutions computed with absorbing layers and the nearly exact solution from the brute force computation.

The reflections depend on the frequency. The asymptotic expansion shows that with $a_{0}^{\mathrm{I}}:=\alpha_{0}^{\mathrm{I}} \Phi^{\mathrm{I}}$, one has $a^{\mathrm{R}}=\alpha^{\mathrm{R}} \Phi^{\mathrm{R}}$, and $\alpha^{\mathrm{R}} \sim R \alpha_{0}^{\mathrm{I}}$, with

$$
R=\left\{\begin{array}{lll}
\text { Smart }: & \frac{R_{S}}{2 \pi \omega}, & R_{S}=\frac{i \sigma k}{4\left(c \varphi^{\prime 2}\right)(r)}, \\
\text { Bérenger }: & \frac{R_{B}}{(2 \pi \omega)^{2}}, & R_{B}=-\frac{\sigma k c^{\prime}(r)}{4 c^{2}(r) \varphi_{+}^{\prime 3}(r)}, \\
\text { Tuned : } & \frac{R_{T}}{(2 \pi \omega)^{2}}, & R_{T}=\frac{\sigma\left(k c^{\prime}(r)+2 c(r) \varphi_{+}^{\prime}(r) \partial_{2}\right)}{4 c^{2}(r) \varphi_{+}^{\prime 3}(r)} .
\end{array}\right.
$$


We choose the velocity $c\left(x_{1}\right)=1 / \cosh \left(x_{1}+1\right)$, for which the eikonal equation for $k=1$, $\varphi^{\prime 2}\left(x_{1}\right)+1=1 / c^{2}$, has the explicit form $\varphi^{I^{\prime}}\left(x_{1}\right)=-\varphi_{+}^{\prime}\left(x_{1}\right)=-\sinh \left(x_{1}+1\right)$. This gives the formulas

$$
R_{S}=i \sigma \frac{\cosh (1+r)}{4 \sinh ^{2}(1+r)}, \quad R_{B}=-\sigma \frac{1}{4 \sinh ^{2}(1+r)}, \quad R_{T}=\sigma \frac{1}{4 \sinh ^{2}(1+r)}\left(1+2 \cosh (1+r) \partial_{2}\right) .
$$

Define

$$
\chi_{\rho, p}(x):=\cos ^{p}\left(\frac{\pi|x|^{2}}{2 \rho^{2}}\right) \mathbf{1}_{|x| \leq \rho}
$$

a $C^{p-1}$ function, compactly supported in the disk of radius $\rho$. The initial data is chosen equal to a polarized wave packet

$u(x, 0)=\chi_{r_{1}, 4}\left(x_{1}\right) \chi_{r_{2}, 2}\left(x_{2}\right) \cos \left(2 \pi \omega\left(-\varphi_{+}\left(x_{1}\right)+k x_{2}\right)\right) \Phi^{\mathrm{I}}, \quad \Phi^{\mathrm{I}}:=\left(c \varphi_{+}^{\prime}, 1+k c\right), r_{2}:=1$.

The asymptotic solution has a wave moving to the right with phase $t+\left(-\varphi_{+}\left(x_{1}\right)+k x_{2}\right)$ and also a wave with amplitude smaller by a factor $1 / \omega$ moving to the left with phase $t-\left(-\varphi_{+}\left(x_{1}\right)+k x_{2}\right)$. When the rightward wave reaches the layer there are reflections. It is the size of those reflections, given by the difference of the computed and nearly exact solutions in $\mathcal{R}$ that we measure.

Equations are solved with a simplified parallel Navier-Stokes solver designed in [11], based on a 4th order discontinuous Galerkin technique in space and a 3rd order TVD (total variation diminishing) Runge-Kutta scheme in time. Fluxes are computed using a characteristic decomposition. The square domain $\mathcal{D}$ is discretized with $\Delta x_{j}=4 / N, N=$ $(400,800,1600,2800)$. The discrete $L^{2}$ norm in space and time in the domain of interest $\mathcal{R}$ is used to measure the error. Figure 2 shows, for various frequencies, the convergence of the numerical error with mesh refinement. The reflection is almost independent of the mesh size for small mesh. This shows that there is a reflection due to the interface and that the reflection is not the result of discretization error.
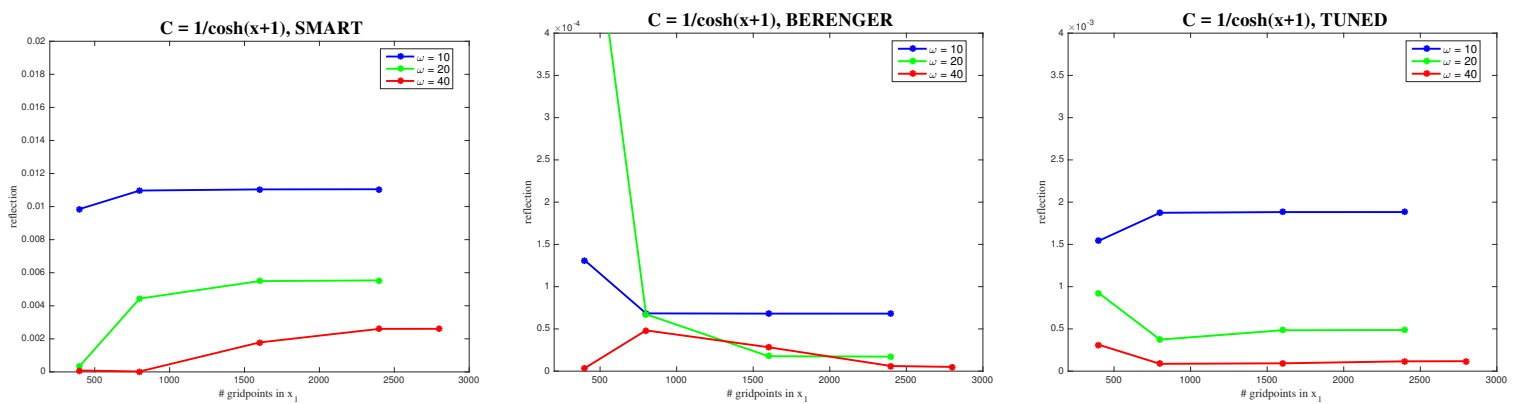

Figure 2: Reflection by a layer as a function of the number of points for 3 values of $\omega$

Figure 3 shows the reflection for small mesh in loglog scale for Bérenger, smart, and tuned layer as a function of the frequency, together with the theoretical expectations. The expected behavior is confirmed. So is the linear dependence on $\sigma$ that we do not display in a figure. For $\omega=10$, the ratio $\left|R_{S} / R_{B}\right|$ predicted by the theory is $2 \pi \omega \cosh (1+r) \sim 161.95$. The numerics yield 162.22 . The ratio $R_{T}\left(u(\cdot, 0) / R_{B}(u(\cdot, 0) \mid\right.$ is about 25 and is independent of the frequency. 

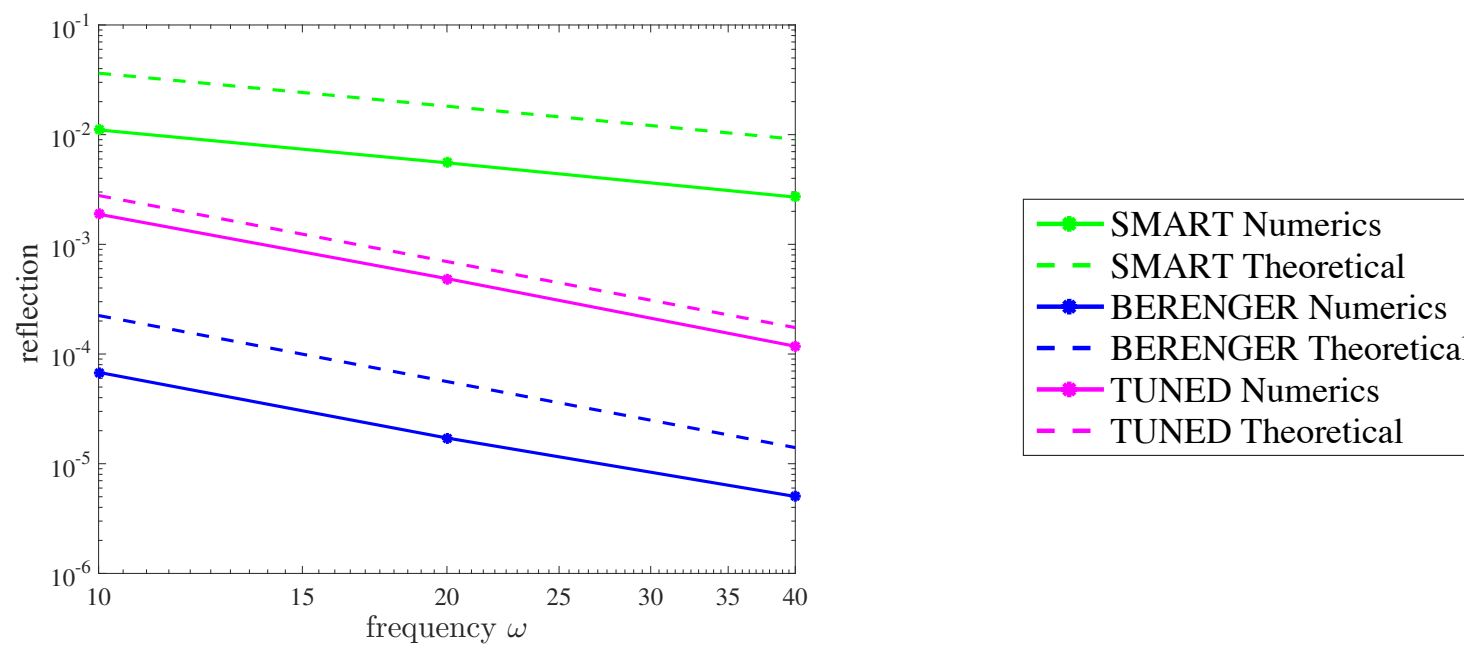

Figure 3: Reflection by smart, tuned and Bérenger layers as a function of the frequency, numerical results and theoretical bounds.

\section{Conclusion}

It is proved by high frequency asymptotics that Bérenger's layer is not perfectly matched for variable coefficients. However the reflection is of order 2 while most smart layers are of order 1. If the wave number $k$ is known, we have introduced a tuned layer with reflections of order 2. The smart and tuned layers are both dissipative in the layer by straightforward energy estimates while Bérenger's layers can be amplifying even in cases where they are perfectly matched. In numerical experiments, Bérenger's layer outperforms the tuned layer by a factor of 20 . The relative error for tuned is already quite small, and the computational cost is half as large.

\section{References}

[1] S. Abarbanel and D. Gottlieb. On the construction and analysis of absorbing layers in CEM. Appl. Numer. Math., 27:331-340, 1998.

[2] S. Abarbanel, D. Gottlieb, and S. Hesthaven. Well-posed perfectly matched layers for advective acoustics. J. Comput. Phys., 154:266-283, 1999.

[3] D. Appelö and T. Colonius. A high-order super-grid-scale absorbing layer and its application to linear hyperbolic systems. Journal of Computational Physics, 228(11):4200-4217, 2009.

[4] D. Appelö, T. Hagström, and G. Kreiss. Perfectly matched layers for hyperbolic systems: General formulation, well-posedness and stability. SIAM J. Appl. Math., $67: 1-23,2006$.

[5] A. Bayliss and E. Turkel. Radiation boundary conditions for wave-like equations. Comm. Pure Appl. Math., 33:707-725, 1980.

[6] E. Bécache, S. Fauqueux, and P. Joly. Stability of perfectly matched layers, group velocities and anisotropic waves. J. Comput. Phys., 188:399-433, 2003. 
[7] E. Bécache and P. Joly. On the analysis of Bérenger's perfectly matched layers for Maxwell's equations. M2AN Math. Model. Numer. Anal., 36:87-119, 2002.

[8] J. P. Bérenger. A perfectly matched layer for the absorption of electromagnetic waves. J. Comput. Phys., 114:185-200, 1994.

[9] J. P. Bérenger. Three-dimensional perfectly matched layer for the absorption of electromagnetic waves. J. Comput. Phys., 127:363-379, 1996.

[10] J.-P. Bérenger. Perfectly matched layer (PML) for computational electromagnetics. Synthesis Lectures on Computational Electromagnetics, 2(1):1-117, 2007.

[11] M. Borrel and J. Ryan. The elastoplast discontinuous Galerkin (EDG) method for the Navier-Stokes equations. Journal of Computational Physics, 231:1-22, 2012.

[12] F. Collino and P. Monk. The perfectly matched layer in curvilinear coordinates. SIAM J. Sci. Comput, 19(6):2061-2090, 1998.

[13] F. Collino and C. Tsogka. Application of the PML absorbing layer model to the linear elastodynamic problem in anisotropic heteregeneous media. Technical report, RR-3471, INRIA, 1998. https://hal.inria.fr/inria-00073219.

[14] B. Engquist and A. Majda. Absorbing boundary conditions for the numerical simulation of waves. Mathematics of Computation, 31:629-651, 1977.

[15] L. Halpern, L. Métivier, and J. Rauch. Perfectly Matched Layers for acoustic waves in 3D heterogeneous media. In Waves 2011: Proceedings of the 10th International Conference on the Mathematical and Numerical Aspects of Waves, pages 587-590. Pacific Institute for the Mathematical Sciences, 2011.

[16] L. Halpern, S. Petit-Bergez, and J. Rauch. The analysis of matched layers. Confluentes Math., 3(2):159-236, 2011.

[17] F. Hu. On absorbing boundary conditions of linearized Euler equations by a perfectly matched layer. J. Comput. Phys., 129:201-219, 1996.

[18] M. Israeli and S. Orszag. Approximation of radiation boundary conditions. J. Comput. Phys., 41:115-135, 1981.

[19] S. Karni. Far-field filtering operators for suppression of reflections of artificial boundaries. SIAM Journal on Numerical Analysis, 33:1014-1047, 1996.

[20] G. Kreiss, B. Krank, and G. Efraimsson. Analysis of stretched grids as buffer zones in simulations of wave propagation. Applied Numerical Mathematics, 107:1-17, 2016.

[21] E. Lindman. Free space boundary conditions for the time dependent wave equation. J. Comput. Phys., 18:66-78, 1975.

[22] A. Mani. Analysis and optimization of numerical sponge layers as a nonreflective boundary treatment. Journal of Computational Physics, 231(2):704-716, 2012.

[23] L. Métivier, R. Brossier, S. Labbé, S. Operto, and J. Virieux. A robust absorbing layer method for anisotropic seismic wave modeling. Journal of Computational Physics, 279:218-240, 2014. 
[24] N. A. Petersson and B. Sjögreen. Wave propagation in anisotropic elastic materials and curvilinear coordinates using a summation-by-parts finite difference method. Journal of Computational Physics, 299:820-841, 2015.

[25] C. M. Rappaport. Interpreting and improving the PML absorbing boundary condition using anisotropic lossy mapping of space. IEEE Trans. Magn., 32:968-974, 1996.

[26] J. Rauch. Hyperbolic Partial Differential Equations and Geometric Optics, volume 133. American Mathematical Soc., 2012.

[27] E. Turkel and A. Yefet. Absorbing PML boundary layers for wave-like equations. Applied Numerical Mathematics, 27(4):533-557, 1998. 